\title{
SAS-6 engineering reveals interdependence between cartwheel and microtubules in determining centriole architecture
}

\author{
Manuel Hilbert ${ }^{1,6,7}$, Akira Noga ${ }^{2,6,7}$, Daniel Frey ${ }^{1,7}$, Virginie Hamel ${ }^{3,6,7}$, Paul Guichard ${ }^{3,6}$, Sebastian H. W. Kraatz ${ }^{1}$, \\ Moritz Pfreundschuh ${ }^{4}$, Sarah Hosner ${ }^{1}$, Isabelle Flückiger ${ }^{3}$, Rolf Jaussi ${ }^{1}$, Mara M. Wieser ${ }^{1}$, Katherine M. Thieltges ${ }^{1}$, \\ Xavier Deupi $^{1,5}$, Daniel J. Müller ${ }^{4}$, Richard A. Kammerer ${ }^{1}$, Pierre Gönczy ${ }^{3,8}$, Masafumi Hirono ${ }^{2,6,8}$ \\ and Michel O. Steinmetz ${ }^{1,8}$
}

Centrioles are critical for the formation of centrosomes, cilia and flagella in eukaryotes. They are thought to assemble around a nine-fold symmetric cartwheel structure established by SAS-6 proteins. Here, we have engineered Chlamydomonas reinhardtii SAS-6-based oligomers with symmetries ranging from five- to ten-fold. Expression of a SAS-6 mutant that forms six-fold symmetric cartwheel structures in vitro resulted in cartwheels and centrioles with eight- or nine-fold symmetries in vivo. In combination with Bld10 mutants that weaken cartwheel-microtubule interactions, this SAS-6 mutant produced six- to eight-fold symmetric cartwheels. Concurrently, the microtubule wall maintained eight- and nine-fold symmetries. Expressing SAS- 6 with analogous mutations in human cells resulted in nine-fold symmetric centrioles that exhibited impaired length and organization. Together, our data suggest that the self-assembly properties of SAS- 6 instruct cartwheel symmetry, and lead us to propose a model in which the cartwheel and the microtubule wall assemble in an interdependent manner to establish the native architecture of centrioles.

The formation of centrosomes in animal cells, as well as that of cilia and flagella in eukaryotic cells, critically depends on the evolutionarily conserved microtubule-based cylindrical centriole. In most species, newly formed centrioles are organized around a nine-fold radially symmetric 'cartwheel' structure. This cartwheel comprises a central hub $\sim 25 \mathrm{~nm}$ in diameter from which nine spokes emanate and connect through a pinhead structure to nine microtubule triplets, which are linked together and constitute a peripheral 'microtubule wall' (reviewed in refs 1,2). A generally acknowledged model posits that the cartwheel acts as a molecular scaffold for the formation of the nine-fold symmetric architecture of centrioles (reviewed in refs 1-7). In its strictest sense, this 'scaffold model' of centriole formation postulates that the nine-fold symmetric cartwheel is formed first and then dictates the assembly of a nine-fold symmetric microtubule wall.
A key prediction of the scaffold model is that changes in cartwheel symmetry should dictate likewise changes in the architecture of the centriole. A central component of the cartwheel is SAS-6, a protein that is essential for cartwheel formation across eukaryotes (reviewed in refs 1,2). In Chlamydomonas, however, despite the absence of cartwheels, $\sim 20 \%$ of cells bearing a deletion of the sole SAS- 6 gene nevertheless assemble centrioles that comprise a circular microtubule wall with a nine-fold symmetry in $\sim 70 \%$ of cases, but with seven-, eight-, ten- or eleven-fold symmetries in the remaining cases ${ }^{8}$. These observations suggest that the cartwheel is critical for proper assembly of the microtubule wall in Chlamydomonas, but also that in this species the centriolar microtubules have an inherent ability to assemble into near-nine-fold symmetrical structures. In contrast, knockdown of SAS-6 proteins in other systems, including human cells ${ }^{9,10}$, results in a failure of centriole formation (reviewed in refs 1,2). Collectively, these

\footnotetext{
${ }^{1}$ Laboratory of Biomolecular Research, Department of Biology and Chemistry, Paul Scherrer Institut, CH-5232 Villigen PSI, Switzerland. ${ }^{2}$ Department of Biological Sciences, University of Tokyo, Tokyo 113-0033, Japan. ${ }^{3}$ Swiss Institute for Experimental Cancer Research (ISREC), School of Life Sciences, Swiss Federal Institute of Technology (EPFL), CH-1015 Lausanne, Switzerland. ${ }^{4}$ Department of Biosystems Science and Engineering, Eidgenössische Technische Hochschule (ETH) Zürich, $\mathrm{CH}-4058$ Basel, Switzerland. ${ }^{5}$ Condensed Matter Theory Group, Paul Scherrer Institut, CH-5232 Villigen PSI, Switzerland. ${ }^{6}$ Present addresses: Roche Pharma Research and Early Development, Molecular Design \& Chemical Biology, Roche Innovation Center Basel, F. Hoffmann-La Roche Ltd, CH-4070 Basel, Switzerland (M.Hilbert); Department of Frontier Bioscience, Hosei University, Koganei, Tokyo 184-8584, Japan (A.N., M.Hirono); University of Geneva, Department of Cell Biology, Sciences III, CH-1211 Geneva, Switzerland (V.H., P.Guichard). ${ }^{7}$ These authors contributed equally to this work.

${ }^{8}$ Correspondence should be addressed to P.Gönczy, M.Hirono or M.O.S. (e-mail: pierre.gonczy@epfl.ch or hirono@hosei.ac.jp or michel.steinmetz@psi.ch)
} 
a

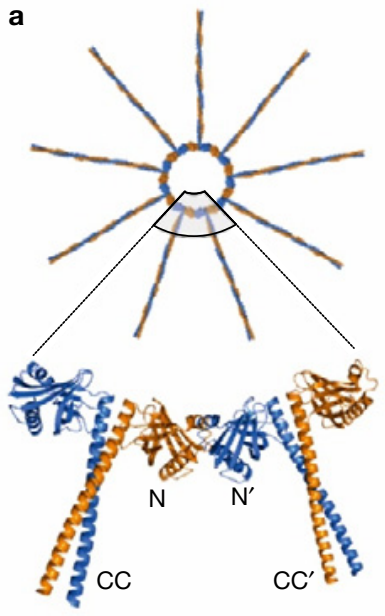

e

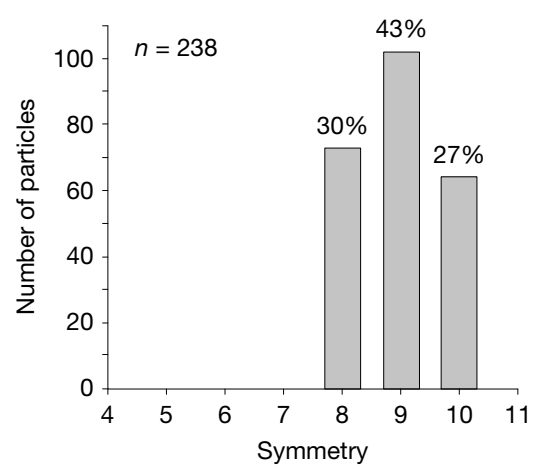

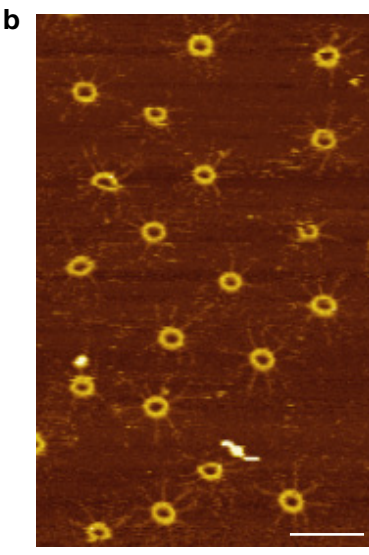
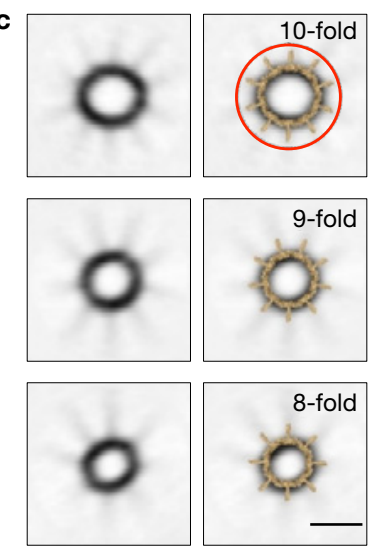
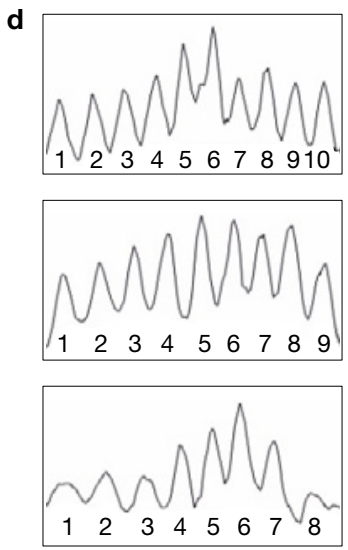

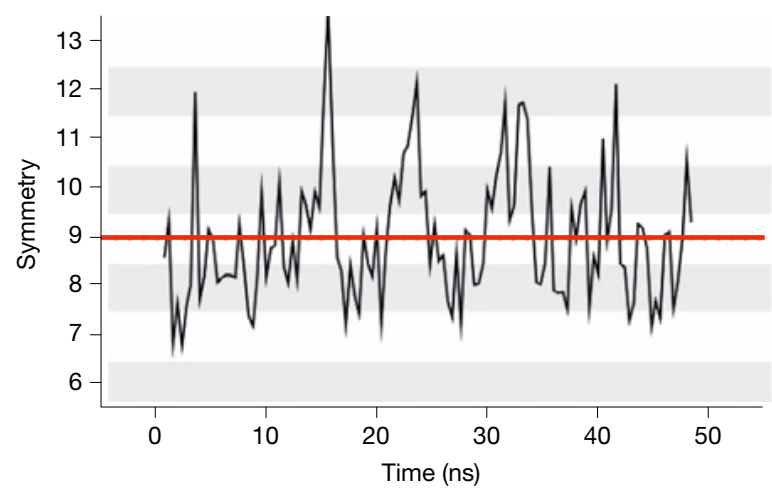

Figure 1 Structural characterization of wild-type CrSAS- 6 N-CC cartwheels. (a) Ribbon representation of a nine-fold symmetric atomic model of the CrSAS- 6 cartwheel based on the crystal structures of the $\mathrm{N}-\mathrm{N}$ dimer and $\mathrm{N}-\mathrm{CC}$ dimer (for details, see ref. 11). The two monomers of the $\mathrm{N}-\mathrm{CC}$ dimer that constitute the fundamental building block of the cartwheel structure are coloured in blue and orange. (b) Representative AFM topograph of selfassembled wild-type CrSAS- $6 \mathrm{~N}-\mathrm{CC}$ cartwheels. Scale bar, $100 \mathrm{~nm}$. (c) Class averages of wild-type CrSAS- $6 \mathrm{~N}-\mathrm{CC}$ cartwheels. Classification and averaging of individual particles taken from AFM topographs reveal the presence of ten- (top), nine- (middle) and eight-fold (bottom) symmetric cartwheels. In

findings emphasize that SAS-6 proteins are important for canonical centriole assembly. However, they also indicate that, at the least in some organisms, additional, cartwheel-independent mechanisms can contribute to determining the native nine-fold symmetric architecture of centrioles, indicating that a strict scaffold model cannot always apply. The exact nature of these additional mechanisms and how they are coordinated with cartwheel-dependent processes is not known.

SAS-6 proteins consist of three domains: an amino-terminal globular head domain (N-domain), a coiled-coil rod domain (CC-domain) and a carboxy-terminal region predicted to be disordered. The CC-domain induces the parallel dimerization of SAS- 6 monomers into homodimers, whereas the $\mathrm{N}$-domain mediates a head-head interaction between SAS- 6 homodimers ${ }^{11-13}$. This arrangement of dimerization interfaces leads to the preferential assembly of 18 SAS-6 monomers into a nine-fold symmetric cartwheel structure in vitro ${ }^{11,12,14}$ (Fig. 1a). In combination with the finding that SAS-6 proteins localize to the cartwheel in centrioles ${ }^{8,15,16}$, these data led the right panels, atomic cartwheel models with the corresponding symmetries have been superimposed to demonstrate the match to the data. The ring in the top-right panel indicates where the radial intensity plots were determined (d). Scale bar, $25 \mathrm{~nm}$. (d) Radial intensity plots of the coiled-coil region of the corresponding averaged wild-type CrSAS- $6 \mathrm{~N}-\mathrm{CC}$ cartwheels shown in c. (e) Frequency of cartwheel symmetries found by AFM in wild-type CrSAS-6 $\mathrm{N}-\mathrm{CC}$ samples. $n$, number of specimens analysed. (f) Predicted fluctuations in cartwheel symmetry during a $50 \mathrm{~ns}$ MD simulation of the wild-type $\mathrm{N}-\mathrm{N}$ dimer. The average symmetry of 8.96 during the course of the simulation is depicted by the red line.

to the conclusion that SAS- 6 constitutes the core of the cartwheel itself $^{16-18}$. This recent progress in our understanding of the structural and functional properties of SAS- 6 proteins provides a unique molecular entry point for modulating cartwheel and centriole architecture by rational engineering, and thus for challenging the scaffold model of centriole assembly. In this study, we introduced mutations in the $\mathrm{N}$-domain of Chlamydomonas SAS- 6 with the aim of changing the symmetry of the resulting cartwheels. The oligomers formed by the various SAS- 6 mutants were characterized in vitro using a combination of biophysical and structural methods, and the impact of symmetrychanging mutations on the architecture of the cartwheel and the microtubule wall was assessed in Chlamydomonas and human cells.

\section{RESULTS}

\section{In vitro characterization of CrSAS-6 oligomers}

Using rotary metal-shadowing electron microscopy (EM), we previously showed that a variant of the Chlamydomonas SAS-6 protein 
Bld12p (CrSAS-6) containing the N- and the CC-domain (N-CC) self-assembles into cartwheel-like oligomers in vitro ${ }^{11}$. To assess the distribution of radial symmetries of CrSAS-6 N-CC cartwheels, we used a high-resolution atomic force microscopy (AFM) method $^{19,20}$ in combination with a reference-free single-particle image analysis. We found that most of the CrSAS- $6 \mathrm{~N}-\mathrm{CC}$ cartwheels exhibited a nine-fold symmetry (Fig. 1b-e); however, the AFM data revealed in addition that CrSAS-6 N-CC can also assemble into cartwheels with eight- and ten-fold symmetries. This heterogeneity suggests that the intramolecular (N-CC) and/or intermolecular (N-N) CrSAS-6 interactions are dynamic to some extent.

To test whether one source of dynamicity stems from the interaction between $\mathrm{N}$-domains from adjacent CrSAS- $6 \mathrm{~N}-\mathrm{CC}$ dimers in the cartwheel, we performed molecular dynamics (MD) simulations using the 2.1- $\AA$-resolution crystal structure of the CrSAS- $6 \mathrm{~N}-\mathrm{N}$ dimer as a starting point ${ }^{11}$. From individual snapshots of the relative arrangement between the $\mathrm{N}$-domains in a $50 \mathrm{~ns} \mathrm{MD}$ trajectory, we extrapolated the radial symmetry of an idealized planar CrSAS-6 $\mathrm{N}-\mathrm{CC}$ cartwheel. As shown in Fig. 1f, the predicted symmetries during the course of the simulation fluctuated between eight- and ten-fold for more than three-quarters of the MD trajectory; in the remaining cases, seven- and eleven- to thirteen-fold symmetries were obtained. The average symmetry over the entire simulation amounted to 8.96 . These results are in good agreement with the distribution of symmetries observed through AFM analysis, demonstrating the dynamic nature of the CrSAS-6 N-N dimer interface.

\section{Engineering of CrSAS- 6 cartwheels with modified symmetries in vitro}

Next, we sought to change the symmetry of the self-assembled CrSAS-6 N-CC cartwheels by rational mutagenesis of its N-domain. The $\mathrm{N}-\mathrm{N}$ interface is characterized by a prominent hydrophobic contact involving Phe145 from one CrSAS-6 N-domain, whose side chain packs against Ile95 and Phe102 of the neighbouring $\mathrm{N}$-domain (Supplementary Fig. 1). Additional interactions are formed by polar and charged residues, including Gln93, Gly94, Lys105, Glu141, Asn143, Asp144, Lys146, Gln147 and His150. We followed three strategies for the mutagenesis. First, we tested the effect of replacing the hydrophobic residues with different hydrophobic ones, which we denoted as the 'hydrophobic contact' approach. In a second 'saltbridge' approach, charged residues were introduced with the aim to either strengthen available salt-bridge interactions or to create new ones. Finally, in a 'covalent linkage' approach, we introduced pairs of cysteine residues to potentially form covalently linked $\mathrm{N}-\mathrm{N}$ dimers under oxidizing conditions.

Following these three strategies in a first round of mutagenesis, we produced 21 variants by introducing single or double mutations in the $\mathrm{N}-\mathrm{N}$ dimer (CrNN1 to CrNN21; Supplementary Table 1). To assess the stabilities of 19 of them, we determined their equilibrium dissociation constant, $K_{\mathrm{d}}$, by analytical ultracentrifugation and isothermal titration calorimetry (AUC and ITC, respectively; for $K_{\mathrm{d}}$ values in the micromolar range) or fluorescence polarization (FP; for $K_{\mathrm{d}}$ values in the sub-micromolar range). As shown in Fig. 2a, half of the mutants were stabilized two- to five-fold compared with the wild type (see also Supplementary Table 1); the remaining mutants weakened or abrogated the $\mathrm{N}-\mathrm{N}$ interaction, and were thus not considered further.
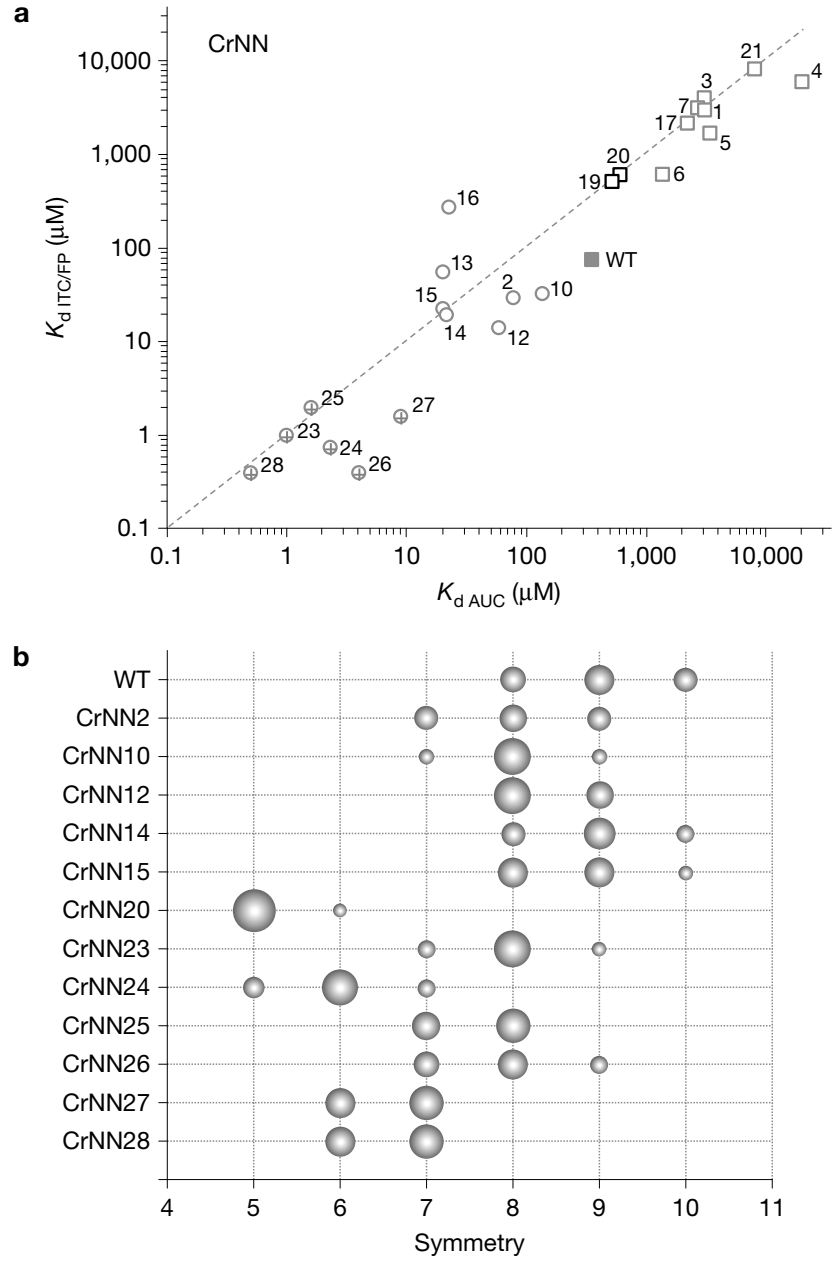

Figure 2 Biophysical and structural characterization of CrSAS-6 N-N and $\mathrm{N}-\mathrm{CC}$ dimer mutants. (a) Stabilities of the $\mathrm{N}-\mathrm{N}$ dimer variants produced in this study assessed by both AUC and ITC or FP. The $K_{d}$ values determined by AUC are indicated on the $x$ axis; the $K_{d}$ values determined by either ITC or FP are indicated on the $y$ axis. Grey open squares and circles highlight destabilizing and stabilizing mutants, respectively, relative to the wild type (WT; filled square). Grey open circles with crosses and black open squares represent data points obtained for the combined mutants and for the mutants following the covalent linkage approach (probed under reducing buffer conditions), respectively. See also Supplementary Table 1. Data points obtained by FP, AUC and ITC represent the average from five, at least two and one independent measurements, respectively. For s.d. values, see Supplementary Table 1. (b) Determination of cartwheel symmetries of selected CrSAS- $6 \mathrm{~N}-\mathrm{CC}$ dimer variants by AFM or EM and subsequent single-particle image analysis. The areas of the grey circles are proportional to the percentage of observed symmetry species (see also Table 1 and Supplementary Fig. 3).

Two out of the three cysteine mutants, CrNN18 and CrNN20, resulted in covalently linked dimers (Supplementary Fig. 1e).

In a second round of mutagenesis, selected stabilizing mutations generated with the 'hydrophobic contact' or the 'salt-bridge' approach were combined into six additional variants ( $\mathrm{CrNN} 23$ to CrNN28; Supplementary Table 1). As shown in Fig. 2a and Supplementary Fig. 1f, the combination of mutations further enhanced the stability of all six mutants by two orders of magnitude when compared with the wild-type $\mathrm{N}-\mathrm{N}$ dimer, resulting in $K_{\mathrm{d}}$ values in the low- to submicromolar range. To further characterize the stabilized variants, we 
Table 1 Symmetries of mutant CrSAS-6 N-CC cartwheels.

\begin{tabular}{|c|c|c|c|}
\hline $\begin{array}{l}\text { CrSAS-6 } \\
\text { variant }\end{array}$ & Mutations & AFM/EM & X-ray \\
\hline WT & - & $\begin{array}{l}8,9,10^{*} \\
(31 \%, 43 \%, 27 \%)\end{array}$ & $9.6 ; 9.7 ; 10.1$ \\
\hline CrNN2 & F145W & $\begin{array}{l}7,8,9^{*} \\
(28 \%, 40 \%, 31 \%)\end{array}$ & $7.1 ; 6.4$ \\
\hline CrNN10 & Q93E & $\begin{array}{l}7,8,9 * \\
(15 \%, 73 \%, 12 \%)\end{array}$ & ND \\
\hline CrNN12 & Q93E K146R & $\begin{array}{l}7,8^{*} \\
(63 \%, 37 \%)\end{array}$ & 8.3 \\
\hline CrNN14 & G94E Q147K & $\begin{array}{l}8,9,10^{*} \\
(28 \%, 53 \%, 19 \%)\end{array}$ & ND \\
\hline CrNN15 & G94D Q147R & $\begin{array}{l}8,9,10^{*} \\
(47 \%, 46 \%, 7 \%)\end{array}$ & ND \\
\hline CrNN18 & K105C F145C & ND & 15.9 \\
\hline CrNN20 & 195C K146C & $\begin{array}{l}5,6^{\dagger} \\
(47 \%, 46 \%, 7 \%)\end{array}$ & $9.5 ; 9.6 ; 10.2$ \\
\hline CrNN23 & $\begin{array}{l}\text { Q93E G94D } \\
\text { K146R Q147R }\end{array}$ & $\begin{array}{l}7,8,9^{\dagger} \\
(17 \%, 72 \%, 12 \%)\end{array}$ & $6.2 ; 7.5$ \\
\hline CrNN24 & $\begin{array}{l}\text { Q93E F145W } \\
\text { K146R }\end{array}$ & $\begin{array}{l}5,6,7^{*, \dagger} \\
(20 \%, 65 \%, 16 \%)\end{array}$ & 5 \\
\hline CrNN25 & $\begin{array}{l}\text { G94D F145W } \\
\text { Q147R }\end{array}$ & $\begin{array}{l}7,8^{\dagger} \\
(53 \%, 47 \%)\end{array}$ & 7.8 \\
\hline CrNN26 & $\begin{array}{l}\text { G94E F145W } \\
\text { Q147K }\end{array}$ & $\begin{array}{l}7,8,9^{\dagger} \\
(32 \%, 49 \%, 19 \%)\end{array}$ & $6.3 ; 6.8$ \\
\hline CrNN27 & Q93E F145W & $\begin{array}{l}6,7^{\dagger} \\
(40 \%, 60 \%)\end{array}$ & $6.3 ; 7$ \\
\hline CrNN28 & $\begin{array}{l}\text { Q93E G94D } \\
\text { F145W K146R } \\
\text { Q147K }\end{array}$ & $\begin{array}{l}6,7^{\dagger} \\
(39 \%, 61 \%)\end{array}$ & $6.3 ; 6.7$ \\
\hline
\end{tabular}

*Symmetry of the indicated CrNN N-CC variant determined on the basis of AFM data. Symmetry of the indicated $\mathrm{CrNN} \mathrm{N-CC}$ variant determined on the basis of negative-stain EM data. ND, not determined. X-ray column: Symmetries inferred from the crystal structures of the corresponding $\mathrm{CrN}-\mathrm{N}$ dimer variants.

solved several high-resolution structures of representative $\mathrm{N}-\mathrm{N}$ dimer mutants by X-ray crystallography, which confirmed the underlying design strategies in most of the cases (Supplementary Fig. 2 and Supplementary Table 2).

To test the effect of mutations on the symmetry of CrSAS-6 cartwheels in vitro, we transferred $13 \mathrm{~N}$-domain mutations into the CrSAS-6 N-CC construct. The structures formed by the various CrSAS-6 N-CC mutants were analysed either by AFM or negativestaining EM followed by single-particle image analysis. With the exception of CrNN18, all the remaining 12 mutants assembled into cartwheel structures (Supplementary Fig. 3). CrNN14 and CrNN15, which exhibited an increased stability by one order of magnitude compared with the wild type, retained preferentially a nine-fold symmetry (Fig. $2 \mathrm{~b}$ and Table 1). However, and remarkably, the remaining ten mutants populated preferentially radial symmetries that were notably altered from that of the wild type, ranging between five- and eight-fold (Fig. $2 \mathrm{~b}$ and Table 1). Inspection of the crystal structures of the corresponding $\mathrm{N}-\mathrm{N}$ dimer mutants (Supplementary Fig. 2) indicated that the observed changes in symmetries could be explained by the altered relative orientation between neighbouring monomers in the $\mathrm{N}-\mathrm{N}$ dimers. These results demonstrate the feasibility of changing the symmetry of CrSAS-6 cartwheels in vitro.

\section{Impact of CrSAS-6 mutations on Chlamydomonas centrioles}

To test the impact of symmetry-changing $\mathrm{N}$-domain mutations in fulllength CrSAS- 6 on the architecture of centrioles in Chlamydomonas, we selected mutations CrNN2, CrNN15 and CrNN26, which in vitro led to a substantial extent to the assembly of cartwheels with eightfold symmetries, and CrNN24, which preferentially forms six-fold symmetric structures. Plasmid constructs containing the full-length CrSAS-6 mutant gene tagged with $\mathrm{C}$-terminal 3xHA tags were introduced into a Chlamydomonas strain that lacks the CrSAS-6 gene $^{8}$ (bld12). Addition of such a tag does not affect the architecture of the cartwheel and the centriole in a significant manner, as all cartwheels and centrioles present in the CrSAS-6-3xHA strain exhibited nine spokes and nine triplets, respectively ${ }^{8,12}$ (Fig. 3a,b and Supplementary Fig. 4a).

Western-blot analysis and immunofluorescence microscopy showed that the expression levels were mildly elevated for all four mutants compared with that of CrSAS- 6 in the wild type and that all of them localized correctly to centrioles (basal bodies; Supplementary Fig. $4 \mathrm{~b}, \mathrm{c})$. The fraction of flagellated cells in the mutant strains was assessed as an indicator of function in centriole assembly. As reported previously ${ }^{8,12}$, only $\sim 10 \%$ of the bld 12 cells formed flagella, reflecting severe defects in centriole formation in the absence of CrSAS-6 (Supplementary Fig. 4d). In contrast, bld12 strains expressing wildtype or mutant CrSAS- 6 rescued the bld 12 phenotype and led to the assembly of functional centrioles to a significant degree.

We then assessed the effect of the four mutants on the symmetry of centrioles by counting the number of circularly arranged microtubule triplets in EM micrographs of nucleo-flagellar apparatus (NFAp)derived basal body cross-sections (Fig. 3a and Supplementary Fig. 4e). As previously reported ${ }^{8}$, most centrioles in bld 12 cells were fragmented. However, $\sim 20 \%$ of cells contained microtubule walls that exhibited a nine-fold radial symmetry in $\sim 70 \%$ of cases, with the remaining ones exhibiting seven-, eight-, ten- or eleven-fold symmetries $^{8}$ (Fig. 3b). Such microtubule triplet-number aberrations were fully rescued by expression of wild-type CrSAS-6. In contrast to the expectation from the in vitro results, CrNN2, CrNN15 and CrNN26 centrioles still harboured nine microtubule triplets in most cases (Fig. 3b). However, the situation differed in the case of CrNN24, which exhibited both nine- and eight-fold symmetric centrioles (Fig. 3b). The difference in phenotype observed with CrNN24 cannot be attributed solely to an increase in $\mathrm{N}-\mathrm{N}$ interaction stability because CrNN24 and CrNN26 N-domain mutants exhibited similar pairwise $K_{\mathrm{d}}$ values in the $1 \mu \mathrm{M}$ range (Fig. $2 \mathrm{a}$ ).

We next investigated whether the symmetry of the corresponding cartwheels was affected. We conducted this analysis with CrNN24 and CrNN26, as these two mutants populate six- and eight-fold symmetric cartwheels in vitro, respectively, yet exhibit similar $K_{\mathrm{d}}$ values for their $\mathrm{N}-\mathrm{N}$ interaction, so that a potential impact on cartwheel symmetry could be distinguished from one stemming merely from a stabilization effect. Cartwheel symmetries were examined by counting the number of spokes emanating from the central hub in EM micrographs of crosssections of the proximal region of centrioles. In the wild type and in CrSAS-6-rescued cells, cartwheel-less centrioles were observed in 0 
a

WT
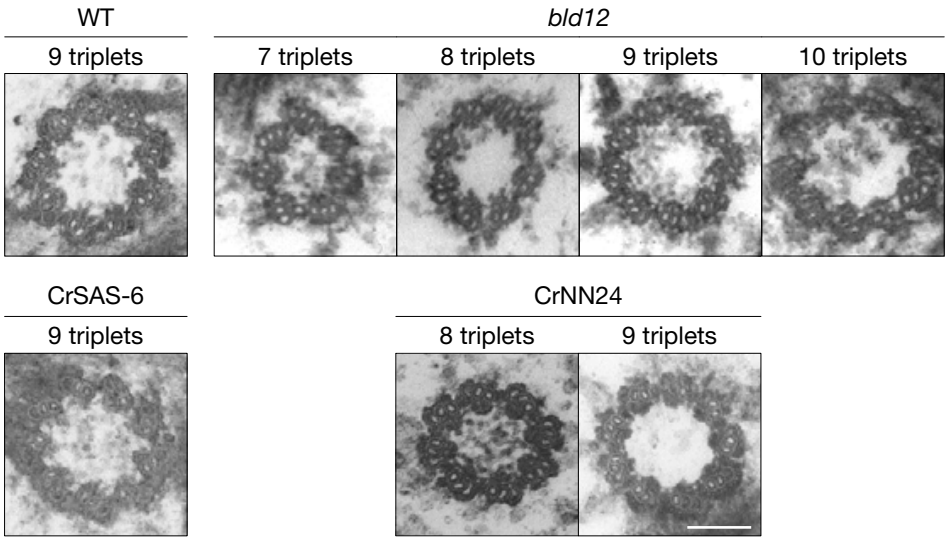

b
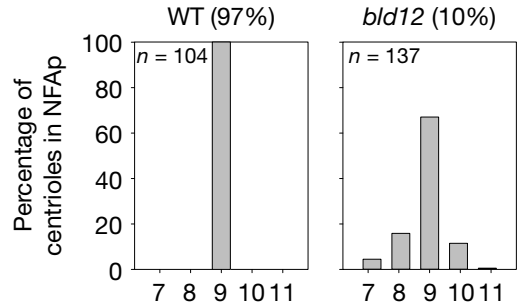

CrSAS-6 (87\%)
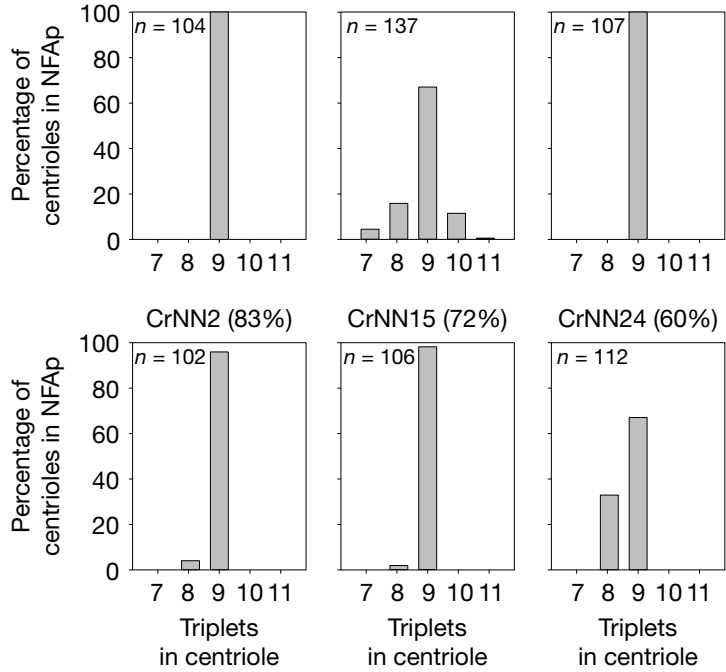

C

CrNN24 (proximal ends, $n=104$ )
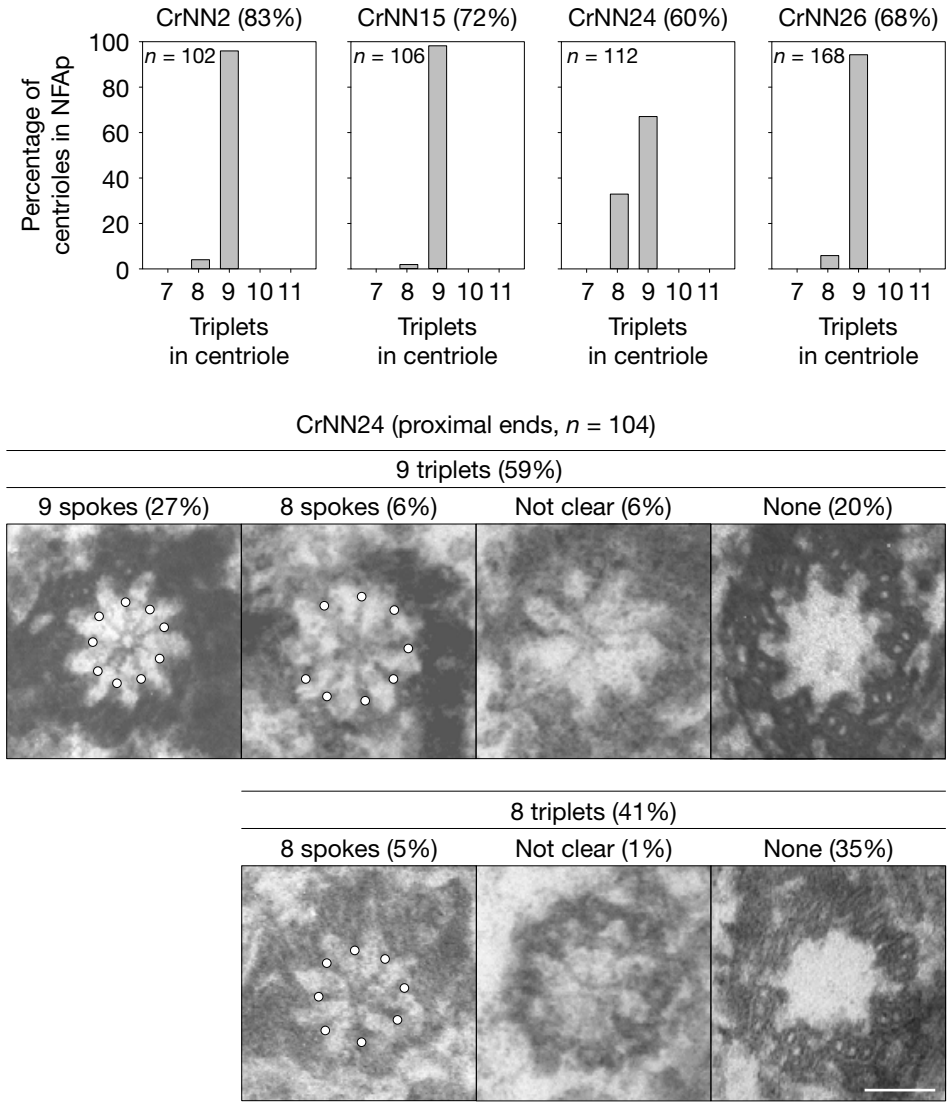

Figure 3 Consequences of expressing CrSAS-6 NN24 in Chlamydomonas. (a) Cross-section electron micrographs of wild-type (WT), bld12, CrSAS-6 and CrNN24 centrioles. (b) Microtubule triplet-number distributions of centrioles in NFAp preparations from WT, bld12, CrSAS-6-, CrNN2-, CrNN15-, CrNN24- and CrNN26-expressing cells. Percentages of flagellated cells in these strains (Supplementary Fig. 4d) are indicated in parentheses. (c) Cross-section electron micrographs of the proximal ends of CrNN24 centrioles. Images of nine- and eight-triplet centrioles were classified into four groups: those containing cartwheels with nine spokes, eight spokes, cartwheels with an unclear number of spokes (Not clear), and no detectable cartwheels (None). Percentages of centrioles obtained by $\mathrm{EM}$ in these classes are indicated in parentheses. Spokes are highlighted with white dots. $n$, number of centrioles analysed by EM. Scale bars, $100 \mathrm{~nm}$. 
a

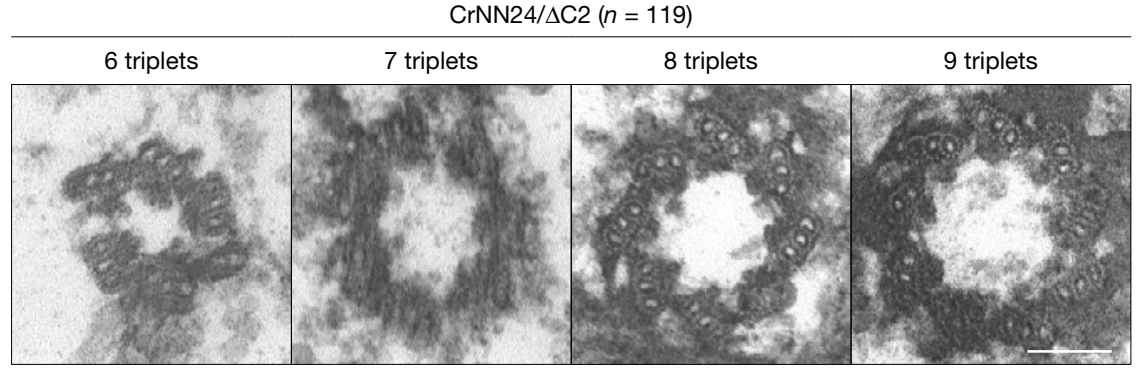

b

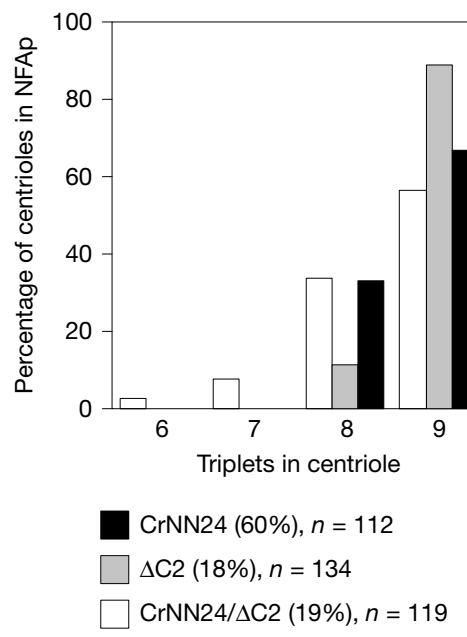

d

CrNN24/AC2 proximal ends $(n=96)$

\begin{tabular}{|c|c|c|c|c|c|}
\hline & \multicolumn{3}{|c|}{ Triplets in centriole } & \multirow[b]{2}{*}{ Total } \\
\hline & & 7 & 8 & 9 & \\
\hline \multirow{4}{*}{ 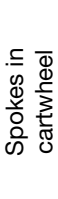 } & 6 & 2 & 1 & 1 & $4(31 \%)$ \\
\hline & 7 & 0 & 1 & 3 & $4(31 \%)$ \\
\hline & 8 & 0 & 1 & 4 & $5(38 \%)$ \\
\hline & 9 & 0 & 0 & 0 & $0(0 \%)$ \\
\hline \multicolumn{2}{|c|}{ Not clear } & 1 & 15 & 28 & \\
\hline \multicolumn{2}{|c|}{ No cartwheel } & 0 & 13 & 26 & \\
\hline
\end{tabular}

c

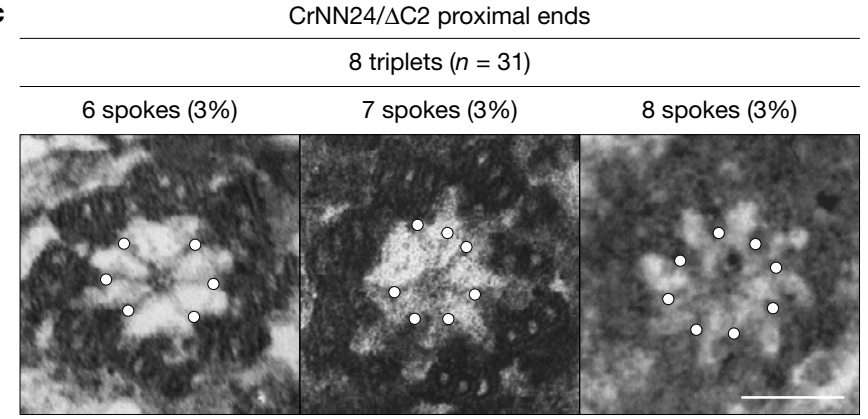

Figure 4 Consequences of co-expressing Bld10p truncations and CrSAS-6 NN24 in Chlamydomonas. (a) Cross-section electron micrographs of centrioles in cells expressing both CrSAS-6 NN24 and the truncated BId10p

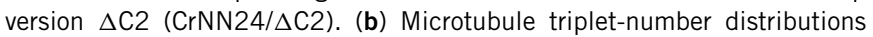
in CrNN24 (black), $\Delta \mathrm{C} 2$ (grey) and $\mathrm{CrNN24/ \Delta C2} \mathrm{(white)} \mathrm{centrioles.}$ Percentages of flagellated cells in these strains (Supplementary Fig. 4d) are indicated in parentheses. (c) Cross-section electron micrographs of the proximal ends of $\mathrm{CrNN} 24 / \Delta \mathrm{C} 2$ centrioles. Spokes are highlighted with white dots. (d) Distributions of microtubule triplet and spoke numbers in CrNN24/ $\Delta$ C2 centrioles. Ninety-six cross-section electron micrographs of the proximal ends of the $\mathrm{CrNN} 24 / \Delta \mathrm{C} 2$ centrioles were classified into 18 groups based on their number of microtubule triplets, cartwheel spokes and appearance of the cartwheel. Numbers and percentages in these groups are listed. $n$, number of centrioles observed by EM. Scale bars, $100 \mathrm{~nm}$. and $13 \%$ of cases, respectively ${ }^{8}$ (Supplementary Fig. 4a). In contrast, centrioles without cartwheels were often observed in CrNN24 and CrNN26 cells (Fig. 3c and Supplementary Fig. 4f). Most cartwheels present in CrNN24 and CrNN26 centrioles with nine triplets exhibited nine spokes, and in a few cases eight spokes; most of these eighttriplet centriole-containing cartwheels exhibited eight spokes in the case of CrNN24 or it was not clear in the case of CrNN26. These results indicate that the CrNN24 and CrNN26 mutations destabilize the cartwheel. The fact that CrNN24 centrioles lack cartwheels twice as frequently as CrNN26 centrioles (Fig. 3c and Supplementary Fig. 4f) suggests that their mere stabilization is not the only reason for this observation. Our data rather indicate that a match between the symmetry of the cartwheel and that of the microtubule wall is critical for producing the stable, native architecture of centrioles.

\section{Role of the cartwheel-microtubule wall interaction in Chlamydomonas}

We set out to test the effect of truncated variants of Bld10p, a protein that contributes to the cartwheel-microtubule wall connection in Chlamydomonas ${ }^{21}$. Expression of truncated versions of Bld10p missing either larger $\mathrm{N}$-terminal $(\Delta \mathrm{N} 3)$ or $\mathrm{C}$-terminal $(\Delta \mathrm{C} 2)$ parts of the protein in the Bld10p null strain (bld10) causes partial 
detachment of the cartwheel spokes from the microtubule wall and formation of aberrant centrioles, including ones exhibiting an eightfold symmetry ${ }^{21}$. We constructed two strains expressing full-length CrSAS-6 containing the CrNN24 mutations together with $\Delta \mathrm{C} 2$ or $\triangle \mathrm{N} 3$ in a CrSAS-6/Bld10p double null background (bld12bld10). In both mutant strains, $\Delta \mathrm{C} 2, \Delta \mathrm{N} 3$ and $\mathrm{CrNN} 24$ were expressed at levels similar to the wild-type proteins (Supplementary Fig. 4b,g,h), and CrNN24 localized to centrioles (Supplementary Fig. 4c). The percentage of flagellated cells in either strain was comparable to that in the single mutants ${ }^{21}$ (Supplementary Fig. 4d). These results document that CrNN24 behaves similarly to wild-type CrSAS- 6 in both Bld10p mutant strains with respect to expression levels, localization and flagella formation.

Inspection of $\mathrm{CrNN} 24 / \Delta \mathrm{C} 2$ centriole cross-sections by $\mathrm{EM}$ revealed that the symmetry-changing CrNN24 mutations still yielded centrioles with eight or nine microtubule triplets in most cases (Fig. 4a,b); this result is similar to the one obtained with the single $\Delta \mathrm{C} 2$ mutant $^{21}$ (Fig. $4 \mathrm{~b}$ and Supplementary Fig. 5a). However, we found that the few remaining $\mathrm{CrNN} 24 / \Delta \mathrm{C} 2$ centrioles exhibited six or seven triplets (Fig. 4a,b). CrNN24/ $\Delta \mathrm{C} 2$ cartwheels, whenever clearly observed (Fig. 4c,d), harboured six, seven or eight, but never nine, spokes, symmetries that are closer to the ones observed in CrNN24 cartwheels obtained in vitro (Fig. 2). This result is in contrast to the $\Delta \mathrm{C} 2$ single mutant, in which cartwheels, whenever observed, exhibited only a nine-fold symmetry ${ }^{21}$ (Supplementary Fig. 5b). In all but one of the CrNN24/ $\Delta \mathrm{C} 2$ cartwheels examined, the number of spokes was less than that of the microtubule triplets, whereas the numbers of spokes matched the numbers of triplets in the $\Delta \mathrm{C} 2$ mutant (Supplementary Table 3). Very similar results were obtained with the CrNN24/ $\Delta \mathrm{N} 3$ double-mutant strain (Supplementary Fig. 5c-h and Supplementary Table 3).

Overall, the results obtained in Chlamydomonas demonstrate that the precise interaction between the cartwheel and the microtubule wall is critical for determining the nine-fold symmetry of centrioles. We also infer from our data that the predominantly nine-fold symmetric microtubule wall is able to select or enforce a nine-fold symmetric cartwheel in CrNN24-expressing strains.

\section{Impact of HsSAS-6 mutations on human centrioles}

To test the impact of substitutions in human cells that are analogous to those in CrNN24, we engineered a mutant version of fulllength HsSAS-6 and fused it to GFP (HsNN24-GFP; Fig. 5a). We found that HsNN24-GFP rescues depletion of endogenous HsSAS-6 as efficiently as wild-type HsSAS-6-GFP (ref. 11; Fig. 5b-e and Supplementary Fig. 6a), demonstrating that the HsNN24 mutations did not impair the function of HsSAS-6. As the cartwheel in human cells cannot be analysed by conventional EM (ref. 22), we conducted immunofluorescence experiments to investigate the presence of the cartwheel in cells expressing HsNN24-GFP, using as a proxy antibodies against STIL, a protein that localizes to the proximal-most region of the emerging centriole ${ }^{23,24}$. We found no difference in the distribution of STIL when comparing cells expressing HsSAS-6-GFP versus HsNN24-GFP (Supplementary Fig. 6b-d), suggesting that the HsNN24 mutations did not inhibit cartwheel formation.

Next, we assessed the architecture of HsNN24 centrioles using immunofluorescence microscopy and EM. We analysed the overall radial organization of the centriole at both proximal and distal ends by stimulated emission depletion (STED) super-resolution microscopy. We conducted this analysis using the proximal and distal centriole markers Cep63 and Cep164, respectively, which both encircle centrioles $^{25-28}$ (Fig. 5f). As shown in Fig. 5g-1, we did not detect major changes in the diameter of Cep63 ring-like structures in cells depleted of endogenous HsSAS-6 and expressing HsNN24-GFP, suggesting that the organization of the proximal region of the centriole is not severely affected. Unexpectedly, in contrast, distal Cep164 ring-like structures were strongly disorganized and larger in such cells than in the control condition.

These data raised the possibility that the architecture of human centrioles is altered by the expression of HsNN24-GFP. To gain further insights into the effect of the HsNN24 mutations on human centrioles, we recorded EM micrographs of cross-sections of isolated HsNN24GFP centrioles and found that, similar to centrioles from control cells, all but one of the HsNN24-GFP centrioles exhibited a microtubule wall with a nine-fold symmetry (Fig. 6a,b and Supplementary Fig. 6e; $n=7)$. In the remaining case, we observed a completely disorganized microtubule wall with a single intact microtubule triplet (Fig. 6c), indicating that the HsNN24 mutations impact centriole stability, thus resulting in some cells that fail to assemble proper centrioles.

Interestingly, EM micrographs of longitudinal sections of HsNN24-GFP centrioles exhibited a significant reduction in length compared with control centrioles, from $\sim 420$ to $\sim 280 \mathrm{~nm}$ on average (Fig. 6d-f). Such a size reduction may explain the results observed by super-resolution microscopy, because Cep164 distribution may be perturbed as a result of disorganization of the distal ends of such shorter centrioles. To collect additional data on centriole size, we used immunofluorescence with antibodies against glutamylated tubulin and CP110 as a means to estimate centriole length (Fig. 6g). As shown in Fig. $6 \mathrm{~h}-\mathrm{j}$ and Supplementary Fig. $6 \mathrm{f}-\mathrm{h}$, expression of wild-type HsSAS-6-GFP in an HsSAS-6-depleted background resulted in centriole sizes that are similar to wild type, demonstrating that the GFP tag does not interfere with centriole architecture. In contrast, expression of HsNN24-GFP under the same conditions resulted in centrioles that were on average $\sim 30 \%$ shorter (Fig. $6 \mathrm{k}$ ), in good agreement with the EM analysis. Overall, these results suggest that the cartwheel plays an important role in determining the stability and overall architecture of human centrioles.

\section{DISCUSSION}

In this study, we challenged the scaffold model of centriole formation by changing the symmetry of SAS-6 cartwheels in vitro using a structure-guided mutagenesis approach, and then assessing the resulting effects on the architecture of centrioles in Chlamydomonas as well as in human cells. The observation that the symmetry-changing NN24 phenotype obtained in vivo is much less pronounced than in vitro is consistent with the notion that cartwheel-independent mechanisms operate in concert with the cartwheel to determine the nine-fold symmetric structure of centrioles (reviewed in refs 1,2). Remarkably, we found that introducing the NN24 version of CrSAS-6 in truncated Bld $10 \mathrm{p}$ mutant strains resulted in centrioles that exhibited unmatched symmetries of the cartwheel and the microtubule wall. We further found that cartwheels in the double CrSAS-6 NN24/Bld10p mutants exhibited symmetries ranging from six- to eight-fold, 


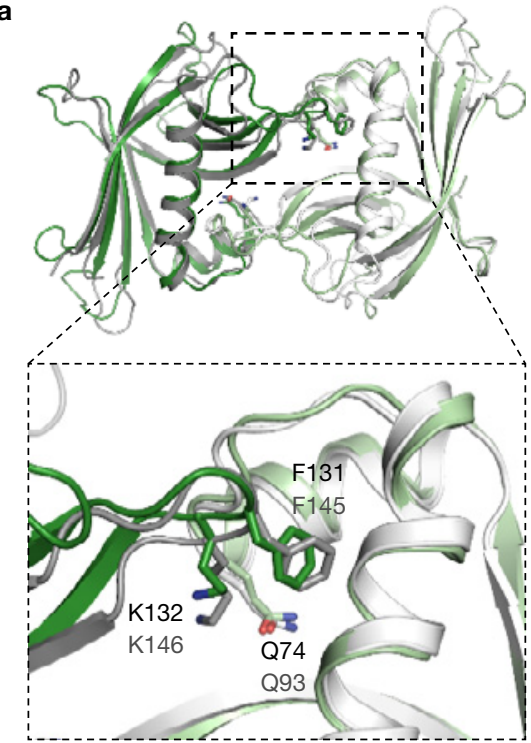

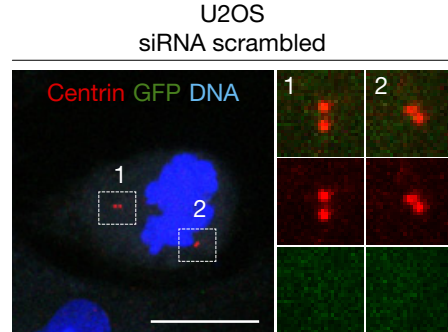

d

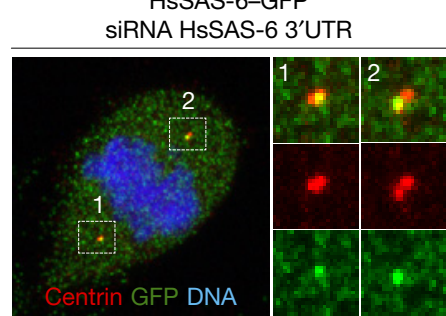

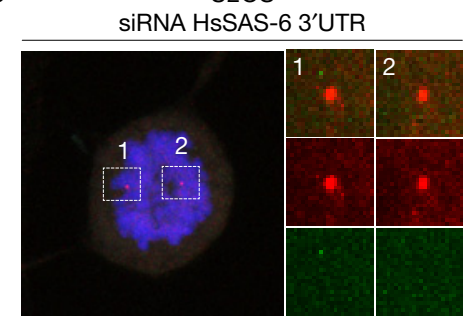

e

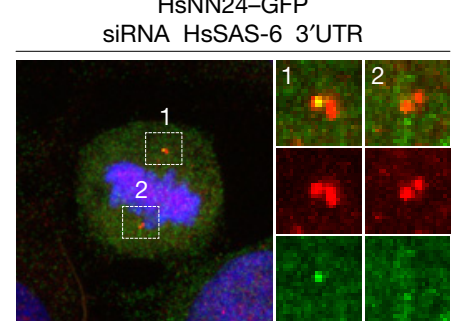

f

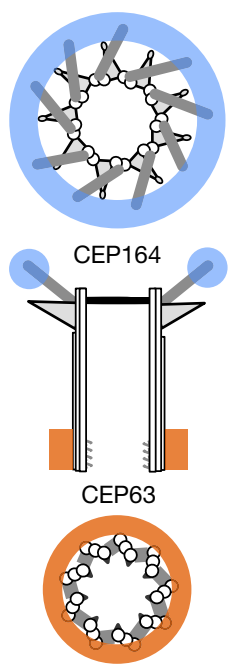

g

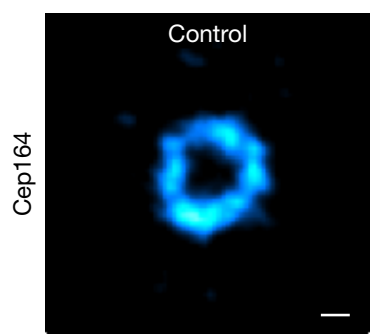

i

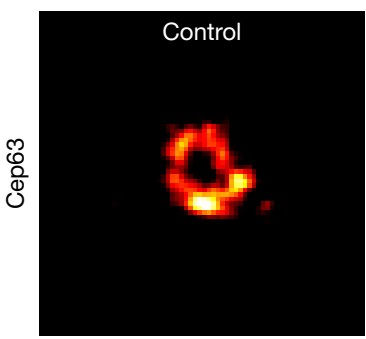

h

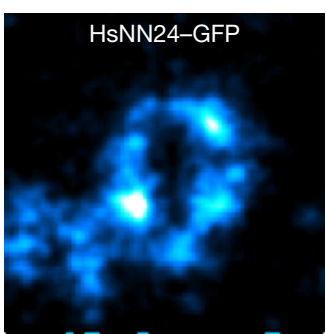

j HsNN24-GFP

k

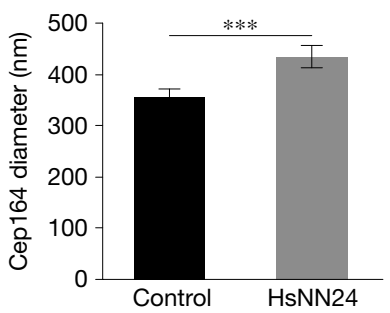

I

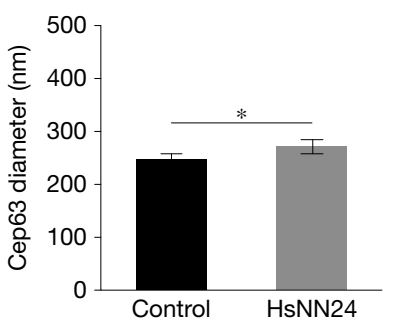

Figure 5 Consequences of expressing HsSAS-6 NN24 in human cells. (a) Structural model of the HsSAS-6 N-N dimer (grey) superimposed onto the crystal structure of the equivalent dimer of CrSAS-6 (green). Inset shows a magnified region, where the residues mutated in the $\mathrm{Cr} / \mathrm{HsNN} 24$ dimer variant are shown as a stick representation. (b-e) Mitotic U2OS (b,c), iU2OS:HsSAS-6-GFP (d) and iU2OS:HsNN24-GFP (e) cells transfected with scrambled siRNAs (b) or siRNAs targeting the $3^{\prime}$ UTR of endogenous HsSAS-6 (c-e), and stained with antibodies against centrin-2 (red) and GFP (green); DNA is in blue. Scale bar, $10 \mu \mathrm{m}$. Insets show magnified views of the delineated centrosomal regions. Note that the centrosomal signal at mitosis seems slightly reduced for HsNN24-GFP (e) compared with HsSAS-6-GFP (d). Data from at least two independent experiments. (f) Schematic representation of a mature centriole; longitudinal view in the centre, cross-sections on top (distal end) and bottom (proximal

which is close to the five- to seven-fold symmetries observed for the CrSAS- 6 cartwheels in vitro, whereas the symmetries of the microtubule wall varied predominantly between eight- and ninefold. The remaining discrepancy between the cartwheel symmetries obtained in vitro and in vivo could be due to the fact that the truncated Bld10p mutants still retain some capacity to interact with and thus influence the symmetry of the cartwheel in vivo ${ }^{21}$. In end). The distal marker Cep164 is represented in blue around the appendages (top), the proximal marker Cep63 in orange (bottom). (g-j) STED images of control U2OS cells $(\mathbf{g}, \mathbf{i})$ or iU2OS:HsNN24-GFP $(\mathbf{h}, \mathbf{j})$ cells depleted of endogenous HsSAS- 6 , and stained with antibodies against Cep164 (g,h) or Cep63 (i,j). Scale bar, $100 \mathrm{~nm}$. (k,l) Ring diameters determined by STED for Cep164 (k) and Cep63 (I). Black columns: control; grey columns: HsNN24-GFP expressed in cells depleted of endogenous HsSAS-6. Data pooled from two independent experiments; average \pm s.d. and $n$ (number of centrioles analysed) were as follows: Cep63 control, $246 \mathrm{~nm} \pm 18$, 14; Cep63 HsNN24, $271 \mathrm{~nm} \pm$ 8, 11; Cep164 control, $355 \mathrm{~nm} \pm 11,21$; Cep164 HsNN24, $435 \mathrm{~nm} \pm 19$, 19. Student's paired $t$-test with a two-tailed distribution: $P=0.0002\left({ }^{* * *}\right)$ for Cep164 and $\left.P=0.038{ }^{*}\right)$ for Cep63. Source data for $\mathbf{k}$ and $\mathbf{I}$ are available in Supplementary Table 4.

addition, post-translational modifications of CrSAS-6 as well as other interacting centriolar proteins may favour the formation of cartwheel symmetries closer to nine in vivo. Overall, these considerations suggest that the self-assembly properties of CrSAS-6 instruct to a large extent cartwheel symmetry in Chlamydomonas. They further suggest that the cartwheel-microtubule wall interaction is critical for establishing a robust nine-fold symmetric architecture of centrioles. 

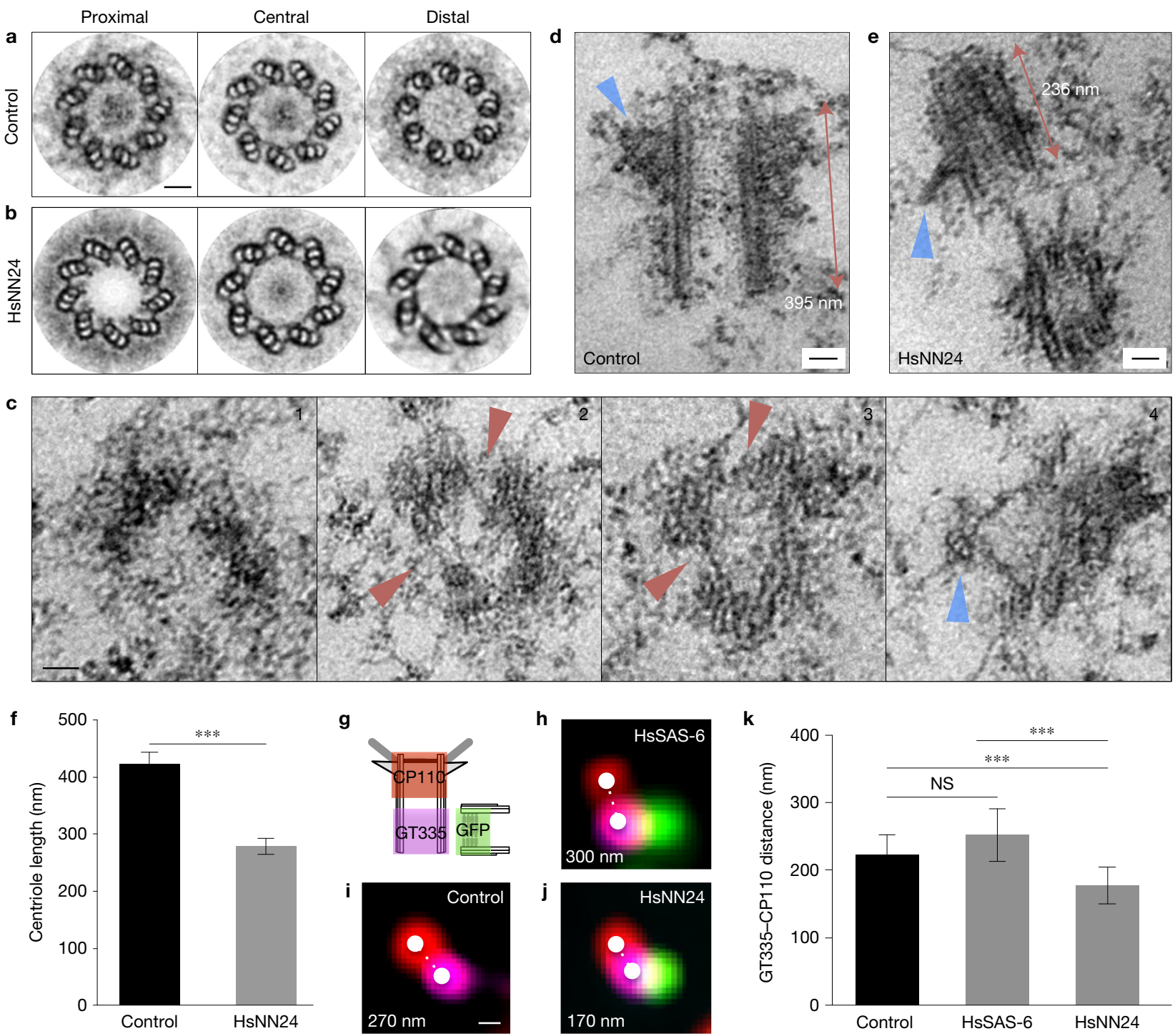

Figure 6 Ultrastructural analysis of human HsSAS-6 NN24 centrioles. $(\mathbf{a}, \mathbf{b})$ Circularized and symmetrized cross-section electron micrographs of centrioles from control U2OS cells (a) or iU2OS:HsNN24-GFP cells depleted of endogenous HsSAS-6 (b). Three regions of the centrioles are shown: proximal, central and distal. Scale bar, $50 \mathrm{~nm}$. (c) Sixty-nanometre-thick serial section electron micrographs of a centriole from a cell expressing HsNN24-GFP and depleted of endogenous HsSAS-6. Note that this centriole is abnormal, with several holes in the periphery (red arrowheads) and only one clearly recognizable microtubule triplet (blue arrowhead). Scale bar, $50 \mathrm{~nm}$. (d,e) Longitudinal electron micrographs of mature centrioles with appendages from the same conditions as in a,b. Note that centrioles from cells expressing HsNN24-GFP (e) are shorter than in the control (d). Scale bars, $50 \mathrm{~nm}$. (f) Quantification of centriolar length from longitudinal electron micrographs; error bars indicate s.d. Black columns correspond to control (average $=423 \mathrm{~nm} \pm 32 ; n=3$ centrioles), grey columns to siRNA targeting HsSAS- 6 (average $=278 \mathrm{~nm} \pm 83 ; n=10$ centrioles). Student's paired $t$-test with a two-tailed distribution: $P=0.015^{(* * *)}$. (g) Schematic

In human cells we did not find evidence that the analogous NN24 mutations in HsSAS-6 impact centriole symmetry; however, we observed a severe impairment of centriole length and overall organization. The observation of different centriolar phenotypes for Chlamydomonas and human NN24 cells is reminiscent of previous representation of a centriole pair with the distal marker $\mathrm{CP} 110$ (red) and the more proximal marker GT335 (monoglutamylated tubulin, magenta) on the parental centriole, as well as GFP (reflecting HsSAS-6-GFP or HsNN24GFP) (green) on the proximal part of the procentriole. (h-j) Representative images of control U20S cells (i), as well as iU20S:HsSAS-6-GFP (h) or iU2OS:HsNN24-GFP (j) cells depleted of endogenous HsSAS-6 and stained with antibodies against CP110 (red), GT335 (magenta) and GFP (green). Scale bar, $200 \mathrm{~nm}$. Insets of centrioles are shown. The distances between the centre of the CP110 and GT335 signals are indicated. (k) Quantification of CP110 to GT335 distances from the experiments illustrated in $\mathbf{h}-\mathbf{j}$; error bars, s.d. Average \pm s.d. and $n$ (number of centrioles analysed) were as follows: control $=224 \mathrm{~nm} \pm 30,11$; HsSAS-6 $=$ $252 \mathrm{~nm} \pm 40,10 ; \mathrm{HsNN} 24=177 \mathrm{~nm} \pm 28,12$. Student's paired $t$-test with a two-tailed distribution: $P=0.08$ (NS, not significant) for HsSAS-6 to control, $P=0.001^{(* *)}$ for HsNN24 to control and $P=4.5 \times 10^{-5}$ ${ }^{(* * *)}$ for HsSAS-6 to HsNN24. Source data for $\mathbf{f}$ and $\mathbf{k}$ are available in Supplementary Table 4. 
Scaffold model

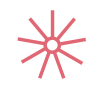

9
$\Rightarrow$

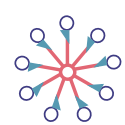

$9 / 9$

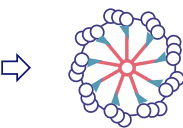

9/9 b Interdependence mode

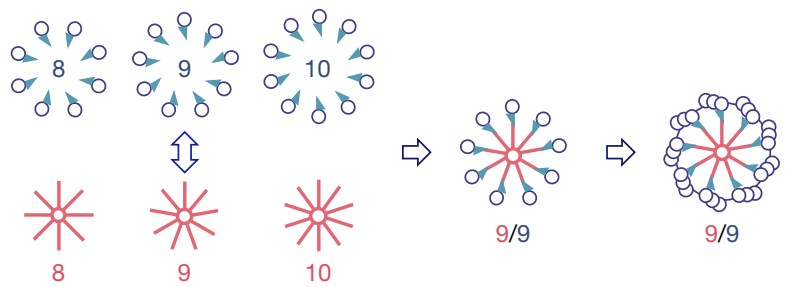

Figure 7 Models of centriole assembly. (a) Scaffold model of centriole assembly. This widely acknowledged model posits that the nine-fold symmetric cartwheel (red) is formed first and functions as a template for the assembly of the nine-fold symmetric microtubule wall (blue). (b) Interdependence model of centriole assembly. Previous data ${ }^{8}$ and the results presented here suggest that both the cartwheel and the microtubule wall can assemble in parallel into transient structures with symmetries ranging predominantly from eight- to ten-fold. The model proposes that the precise match and interplay between a nine-fold symmetric cartwheel and a nine-fold symmetric microtubule wall is essential to produce a stable and native centriole. Notably, the interaction between the cartwheel and the microtubule wall in this model could be mediated, for instance, by the centriolar inner protein BId10p/Cep135, as well as by CPAP and STIL in human cells ${ }^{32,34}$.

or in centrioles that fail to close $\mathrm{e}^{29,30}$. Intriguingly, the observation of aberrant centriole length phenotypes following expression of the HsSAS-6 NN24 mutant is reminiscent of the phenotype incurred on depletion of the cartwheel protein Bld10p/Cep135 in human cells: whereas knocking down Bld10p abrogates centriole assembly in Chlamydomonas ${ }^{21,31}$, this is not the case in human cells, where centrioles depleted of Bld10p/Cep135 readily form, but exhibit a significant reduction in length and a severe disorganization of their distal ends ${ }^{32}$. Notably, a similar reduction of centriole length on knocking down of Bld10p/Cep135 was also observed in Paramecium and Drosophila ${ }^{29,33}$. These results highlight that the nature and degree of impairment in centriole architecture obtained by either mutating or depleting cartwheel proteins vary from one organism to another. They further suggest that the cartwheel as a structural entity or cartwheel proteins play an important role in establishing the native length of centrioles in some cell types.

Collectively, our results have led us to propose a revision of the scaffold model of centriole formation (Fig. 7a). In combination with data from the literature, our findings suggest a mechanism in which the cartwheel and the microtubule wall can form in parallel into assemblies with heterogeneous symmetries, ranging predominantly between eight- and ten-fold. This revised model, which we termed the 'interdependence model' (Fig. 7b), proposes that the two types of centriolar substructure are not stable on their own but that the precise match between a nine-fold symmetric cartwheel and a ninefold symmetric microtubule wall is essential to produce a stable centriole. The in vitro and in vivo approaches developed in the course of this study should enable systematic dissection of the role and relative importance of additional factors that collectively determine the native nine-fold architecture of centrioles.

\section{METHODS}

Methods and any associated references are available in the online version of the paper.

Note: Supplementary Information is available in the online version of the paper

\section{ACKNOWLEDGEMENTS}

We are grateful to V. Oliveri and U. Sauder (University of Basel) for excellent support and access to the electron microscope, and to V. Olieric and M. Wang (Swiss Light Source) for beamline support; we thank R. Kamiya (Gakushuin University) for insightful discussions. The molecular dynamics simulations were performed at the Swiss National Supercomputing Centre and at the high-performance computing facility at the Paul Scherrer Institute, and the EM of human centrioles at the EPFL EM facility (CIME). This work was supported by grants from the ERC (AdG 233335 and AdG 340227; to P.G.), the JSPS KAKENHI (24370079 and 25113503; to M.H.), the Swiss National Centre of Competence in Research 'Molecular Systems Engineering' (to D.J.M. and R.A.K.) and the Swiss National Science Foundation (200021_134521, 31003A_146520 and 310030B_138659; to D.J.M., X.D. and M.O.S., respectively).

\section{AUTHOR CONTRIBUTIONS}

M.Hilbert: designed CrSAS-6 mutants; cloned and produced CrSAS- 6 proteins; designed, performed and analysed biophysical, EM and MD experiments; performed image analysis with AFM and EM data; solved the crystal structure of $\mathrm{N}$-domain mutants; prepared figures and wrote the manuscript. A.N.: designed, performed and analysed the data from Chlamydomonas cell experiments; designed, performed and analysed the EM data from Chlamydomonas cell experiments; prepared figures. D.F.: produced CrSAS- 6 proteins. V.H.: designed, performed and analysed all the data from human cell experiments; prepared figures. P.Guichard: designed, performed and analysed EM experiments on human centrioles; prepared figures. S.H.W.K.: cloned and produced CrSAS-6 proteins; solved crystal structures of $\mathrm{N}$-domain mutants; refined all $\mathrm{N}$-domain mutant crystal structures; prepared figures. M.P.: designed and performed all AFM experiments. S.H.: cloned SAS-6 constructs. I.F.: provided technical assistance for the human cell experiments. R.J.: cloned SAS-6 constructs. M.M.W.: provided technical assistance to the protein purification work. K.M.T.: provided technical assistance to the protein purification work. X.D.: designed CrSAS-6 mutants; designed and supervised all MD experiments. D.J.M.: designed and supervised the AFM experiments. R.A.K.: designed CrSAS-6 mutants; cloned CrSAS- 6 constructs; supervised the production of CrSAS- 6 mutants. P.Gönczy: designed aspects of the study; supervised and analysed the human cell experiments; wrote the manuscript. M.Hirono: designed aspects of the study; supervised and analysed the Chlamydomonas experiments; wrote the manuscript. M.O.S.: designed and supervised the study; supervised and analysed the biophysical and structural data; wrote the manuscript.

\section{COMPETING FINANCIAL INTERESTS}

The authors declare no competing financial interests.

Published online at http://dx.doi.org/10.1038/ncb3329

Reprints and permissions information is available online at www.nature.com/reprints

1. Gönczy, P. Towards a molecular architecture of centriole assembly. Nat. Rev. Mol Cell Biol. 13, 425-435 (2012).

2. Hirono, M. Cartwheel assembly. Phil. Trans. R. Soc. B 369, 20130458 (2014).

3. Strnad, P. \& Gönczy, P. Mechanisms of procentriole formation. Trends Cell Biol. 18 389-396 (2008).

4. Azimzadeh, J. \& Marshall, W. F. Building the centriole. Curr. Biol. 20, R816-R825 (2010).

5. Nigg, E. A. \& Raff, J. W. Centrioles, centrosomes, and cilia in health and disease. Cell 139, 663-678 (2009).

6. Bornens, M. The centrosome in cells and organisms. Science 335, 422-426 (2012).

7. Jana, S. C., Marteil, G. \& Bettencourt-Dias, M. Mapping molecules to structure: unveiling secrets of centriole and cilia assembly with near-atomic resolution. Curr. Opin. Cell Biol. 26, 96-106 (2014).

8. Nakazawa, Y., Hiraki, M., Kamiya, R. \& Hirono, M. SAS-6 is a cartwheel protein that establishes the 9-fold symmetry of the centriole. Curr. Biol. 17, 2169-2174 (2007).

9. Strnad, P. et al. Regulated HsSAS-6 levels ensure formation of a single procentriole per centriole during the centrosome duplication cycle. Dev. Cell 13, 203-213 (2007).

10. Kleylein-Sohn, J. et al. Plk4-induced centriole biogenesis in human cells. Dev. Cell 13, 190-202 (2007).

11. Kitagawa, D. et al. Structural basis of the 9-fold symmetry of centrioles. Cell 144, 364-375 (2011).

12. van Breugel, M. et al. Structures of SAS-6 suggest its organization in centrioles. Science 331, 1196-1199 (2011). 
13. Qiao, R., Cabral, G., Lettman, M. M., Dammermann, A. \& Dong, G. SAS-6 coiled-coil structure and interaction with SAS- 5 suggest a regulatory mechanism in $C$. elegans centriole assembly. EMBO J. 31, 4334-4347 (2012).

14. van Breugel, M., Wilcken, R., McLaughlin, S. H., Rutherford, T. J. \& Johnson, C. M. Structure of the SAS-6 cartwheel hub from Leishmania major. eLife 3, e01812 (2014).

15. Keller, D. et al. Mechanisms of HsSAS- 6 assembly promoting centriole formation in human cells. J. Cell Biol. 204, 697-712 (2014).

16. Guichard, P. et al. Native architecture of the centriole proximal region reveals features underlying its 9-fold radial symmetry. Curr. Biol. 23, 1620-1628 (2013).

17. Culver, B. P., Meehl, J. B., Giddings, T. H. Jr \& Winey, M. The two SAS-6 homologs in Tetrahymena thermophila have distinct functions in basal body assembly. Mol. Biol. Cell 20, 1865-1877 (2009).

18. Guichard, P. et al. Cartwheel architecture of Trichonympha basal body. Science 337, 553 (2012).

19. Pfreundschuh, M., Martinez-Martin, D., Mulvihill, E., Wegmann, S. \& Muller, D. J. Multiparametric high-resolution imaging of native proteins by force-distance curvebased AFM. Nat. Protoc. 9, 1113-1130 (2014)

20. Pfreundschuh, M., Alsteens, D., Hilbert, M., Steinmetz, M. O. \& Muller, D. J. Localizing chemical groups while imaging single native proteins by high-resolution atomic force microscopy. Nano. Lett. 14, 2957-2964 (2014).

21. Hiraki, M., Nakazawa, Y., Kamiya, R. \& Hirono, M. Bld10p constitutes the cartwheelspoke tip and stabilizes the 9-fold symmetry of the centriole. Curr. Biol. 17, 1778-1783 (2007)

22. Guichard, P., Chretien, D., Marco, S. \& Tassin, A. M. Procentriole assembly revealed by cryo-electron tomography. EMBO J. 29, 1565-1572 (2010).

23. Arquint, C., Sonnen, K. F., Stierhof, Y. D. \& Nigg, E. A. Cell-cycle-regulated expression of STIL controls centriole number in human cells. J. Cell Sci. 125, 1342-1352 (2012).
24. Sonnen, K. F., Schermelleh, L., Leonhardt, H. \& Nigg, E. A. 3D-structured illumination microscopy provides novel insight into architecture of human centrosomes. Biol. Open. 1, 965-976 (2012).

25. Lukinavicius, G. et al. Selective chemical crosslinking reveals a Cep57-Cep63Cep152 centrosomal complex. Curr. Biol. 23, 265-270 (2013).

26. Lau, L. et al. STED super-resolution microscopy in tissue and in mammalian cells. Proc. Soc. Photo Opt. Instrum. Eng. (Vol. 7910) http://dx.doi.org/10.1117/12. 881221 (2011).

27. Sir, J. H. et al. A primary microcephaly protein complex forms a ring around parenta centrioles. Nat. Genet. 43, 1147-1153 (2011).

28. Brown, N. J., Marjanovic, M., Luders, J., Stracker, T. H. \& Costanzo, V. Cep63 and cep152 cooperate to ensure centriole duplication. PLOS ONE 8, e69986 (2013).

29. Jerka-Dziadosz, M. et al. Basal body duplication in Paramecium: the key role of Bld10 in assembly and stability of the cartwheel. Cytoskeleton 67, 161-171 (2010).

30. Rodrigues-Martins, A. et al. DSAS-6 organizes a tube-like centriole precursor, and its absence suggests modularity in centriole assembly. Curr. Biol. 17, 1465-1472 (2007)

31. Matsuura, K., Lefebvre, P. A., Kamiya, R. \& Hirono, M. Bld10p, a novel protein essential for basal body assembly in Chlamydomonas: localization to the cartwheel, the first ninefold symmetrical structure appearing during assembly. J. Cell Biol. 165, 663-671 (2004).

32. Lin, Y. C. et al. Human microcephaly protein CEP135 binds to hSAS- 6 and CPAP, and is required for centriole assembly. EMBO J. 32, 1141-1154 (2013).

33. Mottier-Pavie, V. \& Megraw, T. L. Drosophila bld10 is a centriolar protein that regulates centriole, basal body, and motile cilium assembly. Mol. Biol. Cell 20, 2605-2614 (2009).

34. Hatzopoulos, G. N. et al. Structural analysis of the G-box domain of the microcephaly protein CPAP suggests a role in centriole architecture. Structure $\mathbf{2 1}$ 2069-2077 (2013). 


\section{METHODS}

Protein production and purification. Chlamydomonas CrSAS-6 (Uniprot ID A9CQL4) N-terminal domain variants (residues 1-159), named CrNN1 to CrNN28, were generated by PCR mutagenesis ${ }^{35}$ and verified by Sanger sequencing. CrSAS- 6 6HR (residues 1-226) and N-CC constructs (residues 1-503) were generated by BamHI/EagI restriction cloning. BamHI restriction is natively present in all constructs; the EagI restriction site was introduced by a silent mutation at the Pro157 nucleotide position of the PSTCm1 vector ${ }^{35}$. All inserts were generated by PCR amplification of the CrNN variants with a primer containing the appropriate restriction sites.

For protein production, expression plasmids were transformed into the Escherichia coli strain BL21(DE3) (Stratagene). Four to six litres of LB medium supplemented with $50 \mu \mathrm{g} \mathrm{ml}^{-1}$ kanamycin was inoculated with an overnight culture. After incubation for $4 \mathrm{~h}$ at $37^{\circ} \mathrm{C}$, protein expression was induced at an $\mathrm{OD}_{600 \mathrm{~nm}}$ of 0.6 by the addition of $0.4 \mathrm{mM}$ isopropyl-beta-D-thiogalactoside (IPTG) overnight at $20^{\circ} \mathrm{C}$.

Cell pellets were lysed by lysozyme treatment and sonication, and resuspended in lysis buffer containing $50 \mathrm{mM}$ Tris- $\mathrm{HCl}, \mathrm{pH} 7.5,500 \mathrm{mM} \mathrm{NaCl}, 10 \mathrm{mM}$ imidazole, complete protease inhibitor (Roche) and $5 \mathrm{mM} \beta$-mercaptoethanol. Cell debris was removed by centrifugation. Supernatants were applied onto a HisTrap FF crude immobilized metal-affinity column (IMAC; loading buffer: $50 \mathrm{mM}$ Tris- $\mathrm{HCl}$, $\mathrm{pH}$ 7.5, $500 \mathrm{mM} \mathrm{NaCl}, 10 \mathrm{mM}$ imidazole, $5 \mathrm{mM} \beta$-mercaptoethanol; washing buffer: same but containing $70 \mathrm{mM}$ imidazole; elution buffer: same but containing $200 \mathrm{mM}$ imidazole). Eluted proteins were further purified on a Superdex S200 16/60 size exclusion column (SEC buffer: $50 \mathrm{mM}$ Tris- $\mathrm{HCl}, \mathrm{pH} 7.5,150 \mathrm{mM}$ $\mathrm{NaCl}, 5 \mathrm{mM} \beta$-mercaptoethanol) according to the manufacturer's instructions (GE Healthcare)

Proteins used for crystallization and AFM imaging were incubated in $20 \mathrm{mM}$ Tris- $\mathrm{HCl}, \mathrm{pH} 7.4,150 \mathrm{mM} \mathrm{NaCl}, 2.5 \mathrm{mM} \mathrm{CaCl}_{2}$ overnight at $4{ }^{\circ} \mathrm{C}$ with thrombin (Sigma-Aldrich) to remove the 6xHis-tag after the first IMAC. Processed proteins were separated from the cleaved His-tags by a second IMAC chromatography step before the final size exclusion chromatography step.

Protein samples were concentrated and the homogeneity and identity of the recombinant proteins were assessed by SDS-PAGE and mass spectral analysis, respectively. The concentration of protein samples was determined by absorption at $280 \mathrm{~nm}$.

Biophysical methods. Sedimentation equilibrium analytical ultracentrifugation (AUC) experiments were performed at $10^{\circ} \mathrm{C}$ at rotor speeds of 24,000 or 32,000 r.p.m. in SEC buffer on a ProteomeLab XL-I analytical ultracentrifuge (Beckman). The protein concentration was $200 \mu \mathrm{M}$ and all CrSAS-6 N-N dimer samples were run at least in duplicates. The data were recorded by absorbance at $280 \mathrm{~nm}$ and with the interference optics of the centrifuge, and processed with the UltraSpin software package (D. Verprintsev, http://www.mrc-lmb.cam.ac.uk/dbv/ ultraspin2). Partial specific volumes were calculated from the amino acid sequence using SEDENTERP (ref. 36)

Isothermal titration calorimetry (ITC) experiments were performed at $10^{\circ} \mathrm{C}$ using an ITC200 calorimeter (Microcal). N-N dimer samples in the syringe (0.6-1.6 mM) were injected stepwise into SEC buffer. The dissociation isotherms were fitted using a nonlinear least-squares minimization method provided with the calorimeter.

Fluorescence polarization (FP) experiments were performed at $20^{\circ} \mathrm{C}$ in a Cary Eclipse fluorescence spectrophotometer (Agilent Technologies) equipped with an automated polarizer $\left(I_{\mathrm{ex}}=285 \mathrm{~nm} ; l_{\mathrm{em}}=345 \mathrm{~nm}\right.$; slit, $20 \mathrm{~nm} ; 5 \mathrm{~s}$ acquisition time). $\mathrm{N}-\mathrm{N}$ dimer samples $(100 \mu \mathrm{M})$ were diluted stepwise into SEC buffer. The voltage of the detector was adjusted to account for the change in tryptophan fluorescence. Each data point represents the average from five independent measurements. The $K_{\mathrm{d}}$ values were determined by fitting the data with a homodimer model using Origin 7 (OriginLab).

Molecular dynamics (MD) simulations. The high-resolution X-ray structure of the CrSAS-6 N-N dimer (Protein Data Bank ID 3Q0Y; residues 14-159, chains $\mathrm{C}$ and $\mathrm{D})$ was subjected to MD simulations using spherical boundary conditions. Using the program VMD (ref. 37), hydrogen atoms were added to the structure, which was then embedded in a sphere of explicit water molecules with a radius of $50 \AA$. Sodium and chloride ions were added to a concentration of $150 \mathrm{mM} \mathrm{NaCl}$, and then additional ions were added to achieve charge neutrality. The system was equilibrated as follows: first, the starting geometry was optimized with 50,000 steps of energy minimization; then, the temperature was increased to $310 \mathrm{~K}$ in $10 \mathrm{~K}$ intervals. At each of these intervals, the system was simulated for 5,000 steps followed by 20,000 steps of energy minimization. After equilibration, a simulation was run for $50 \mathrm{~ns}$, and snapshots of the trajectory were extracted every $0.4 \mathrm{~ns}$ for analysis with AmberTools12 (ref. 38). Temperature was controlled using Langevin dynamics. The simulation was performed in NAMD (ref. 39) with the CHARMM27 force field ${ }^{40}$ and at a time step of $2 \mathrm{fs}$, while constraining all bonds between hydrogen and heavy atoms.

Overlays of the CrSAS-6 N-CC dimer (Protein Data Bank ID code 3Q0X) with the CrSAS- $6 \mathrm{~N}-\mathrm{N}$ dimers obtained from the MD simulations were used to predict the symmetry of putative cartwheels for every snapshot as described previously ${ }^{11,41}$ The symmetries of the resulting CrSAS-6 N-CC cartwheel structures were calculated with a custom-made PyMOL (The PyMOL Molecular Graphics System, Version 1.7.4 Schrödinger, LLC.) script.

Atomic force microscopy (AFM). CrSAS-6 N-CC samples were diluted in adsorption buffer $(300 \mathrm{mM} \mathrm{KCl}, 20 \mathrm{mM}$ Tris- $\mathrm{HCl}, \mathrm{pH} 7.2)$ to reach a final concentration of $60 \mathrm{nM}$. Thirty microlitres of sample was adsorbed for $10 \mathrm{~min}$ onto a freshly cleaved mica surface. The samples were subsequently rinsed five times with imaging buffer $(20 \mathrm{mM}$ Tris- $\mathrm{HCl}, \mathrm{pH} 7.2,150 \mathrm{mM} \mathrm{KCl})$ and characterized by AFM in imaging buffer at room temperature $\left(26^{\circ} \mathrm{C}\right)$ as described previously ${ }^{20}$. Briefly, the AFM (Multimode 8 equipped with a Nanoscope V controller, Bruker) was operated in the 'PeakForceTapping' mode. The AFM was equipped with a $120 \mu \mathrm{m}$ piezoelectric scanner. Rectangular $\mathrm{Si}_{3} \mathrm{~N}_{4}$ cantilevers having a nominal spring constant of $\sim 0.04-0.08 \mathrm{~N} \mathrm{~m}^{-1}$ and a resonance frequency in water of $\sim 35 \mathrm{kHz}$ were chosen (Biolever Mini, Olympus). AFM topographs (frame size $0.5-3 \mu \mathrm{m}$ ) were recorded applying a vertical oscillation frequency of $2 \mathrm{kHz}$ and amplitude of $25 \mathrm{~nm}$ to the AFM cantilever, an imaging force of $45-65 \mathrm{pN}$, a scanning frequency of $0.3-0.8$ line $\mathrm{s}^{-1}$, and recording topographs of $512 \times 512$ or $1,024 \times 1,024$ pixels in size.

Electron microscopy (EM). For negative-staining transmission EM of purified cartwheel-like specimens, CrSAS-6 N-CC samples were diluted to $0.1 \mathrm{mg} \mathrm{ml}^{-1}$ in $50 \mathrm{mM}$ Tris-HCl, $\mathrm{pH} 7.5,150 \mathrm{mM} \mathrm{NaCl}, 2 \mathrm{mM} \beta$-mercaptoethanol and immediately transferred onto freshly glow-discharged carbon-coated copper grids. Grids were washed with buffer and specimens were negatively stained with freshly prepared uranyl acetate $(1 \% \mathrm{wt} / \mathrm{vol})$.

For EM of Chlamydomonas centrioles, nucleo-flagellar apparatus (NFAp), a cytoskeletal complex that contains centrioles, was isolated from Chlamydomonas cells as described previously ${ }^{42}$. The NFAp was prefixed with $2 \%$ glutaraldehyde and $1 \%$ tannic acids in $50 \mathrm{mM}$ sodium phosphate buffer $(\mathrm{pH} 7.2)$ for $1 \mathrm{~h}$ at $0{ }^{\circ} \mathrm{C}$, and postfixed with $1 \% \mathrm{OsO}_{4}$ in phosphate buffer for $1 \mathrm{~h}$. The samples were dehydrated and embedded in EPON 812 resin. Thin sections 70-nm thick were stained with 7\% uranyl acetate and Reynolds' lead citrate, and observed under a transmission EM.

For EM of human centrioles, three $10 \mathrm{~cm}$ dishes containing iU2OS:HsNN24GFP cells were induced with $1 \mu \mathrm{g} \mathrm{ml}^{-1}$ doxycycline for $60 \mathrm{~h}$ while depleting endogenous HsSAS-6 using an siRNA (Life Technology) targeting the 3'UTR of endogenous HsSAS- 6 as described previously ${ }^{11}$. Centrosome isolation was performed as described previously ${ }^{43}$. Briefly, nocodazole and cytochalasin D were added for $1 \mathrm{~h}$ at $37^{\circ} \mathrm{C}$ to depolymerize the cytoskeleton. Cells were washed with cold PBS, then lysed by the addition of $1 \mathrm{mM}$ Hepes $\mathrm{pH} 7.0 ; 0.5 \% \mathrm{NP}-40 ; 0.5 \mathrm{mM} \mathrm{MgCl}_{2}$ and complemented with a protease inhibitor cocktail (Sigma, reference P8340) on ice. Lysed cells were collected and incubated on ice for $10 \mathrm{~min}$ before the addition of DNAse for $1 \mathrm{~h}$ on ice and then centrifuged for $7 \mathrm{~min}$ at $4{ }^{\circ} \mathrm{C}$ at $100 \mathrm{~g}$. The resulting supernatant was loaded on $1 \mathrm{ml}$ of $60 \%$ sucrose, and then centrifuged at $10,000 \mathrm{~g}$ for $10 \mathrm{~min}$. The centrosome fraction was collected at the interphase between the sucrose and the supernatant. EM was performed as described previously ${ }^{16}$. Briefly, samples were fixed in $2 \%$ paraformaldehyde and $1.5 \%$ glutaraldehyde for $60 \mathrm{~min}$ and washed in cold cacodylate buffer $(0.1 \mathrm{M}, \mathrm{pH} 7.4)$. The sample was then postfixed with $1 \%$ osmium tetroxide at room temperature. Finally, the sample was stained in $2 \%$ uranyl acetate and dehydrated in a graded alcohol series $(2 \times 50 \%, 1 \times 70 \%, 1 \times 90 \%$, $1 \times 95 \%, 2 \times 100 \%$ ), before embedding in Durcupan resin and polymerization at $65^{\circ} \mathrm{C}$ overnight.

Single-particle image analysis. Images of CrSAS-6 N-CC cartwheels obtained by AFM (between 2 and 9 topographs per sample) or negative-stain EM (between 20 and 30 micrographs per sample) were picked by hand using the program Bshow from the Bsoft software package ${ }^{44}$. Only particles that exhibited a closed and circular, not distorted, cartwheel-like structure were picked. Individual particles from various micrographs were stacked, centred and subsequently sorted into classes by reference-free classification using the SPIDER software package ${ }^{45}$. A total of $105-1,854$ particles were classified in up to 5 classes per CrSAS-6 N-CC variant (see also Supplementary Fig. 4).

X-ray crystallography and structural analysis. Crystals of mutant CrSAS-6 N-N dimer variants were grown with the sitting drop vapour diffusion method and appeared under similar conditions as the wild-type protein ${ }^{11}$. However, finescreening of crystallization conditions was necessary and was performed by mixing $200 \mathrm{nl}$ protein solution with $200 \mathrm{nl}$ mother liquor using a Phoenix robot (Art Robin Instruments). 
Diffraction data were collected under cryogenic conditions (100 K) at the X06DA and X06SA macromolecular crystallography beamlines of the Swiss Light Source (Paul Scherrer Institut). For cryoprotection, the crystals were stepwise transferred to mother liquor supplemented with $20 \% \mathrm{vol} / \mathrm{vol}$ glycerol, and flash frozen in liquid nitrogen. Diffraction data were integrated with XDS and scaled with XSCALE (ref. 46). All structures were solved by molecular replacement using $\operatorname{Phaser}^{47}$ with the structure of the monomer of the wild-type CrSAS- $6 \mathrm{~N}$-terminal domain (Protein Data Bank ID 3Q0Y) as a search model.

Manual model building was carried out using COOT (ref. 48) and refined against the native data with PHENIX.refine (ref. 49). PyMOL was used for image preparation. Modelling of higher-order CrSAS-6 oligomers from the various crystal structures was carried out as previously described ${ }^{11,41}$. The model of the HsSAS-6 $\mathrm{N}-\mathrm{N}$ dimer was generated using SWISS-MODEL with default setting $\mathrm{s}^{50}$. The SAS-6 $\mathrm{N}-\mathrm{N}$ dimer of Danio rerio (PDB ID 2Y3V) was used as a template, as it shows $69 \%$ sequence identity with the one of HsSAS-6.

Chlamydomonas strains. The Chlamydomonas reinhardtii wild-type strain CC124 and the cell-wall-less mutant $c w 92$ were obtained from the Chlamydomonas Resource Center (University of Minnesota). The mutant bld12-1 (referred to as bld12) has been previously described ${ }^{8}$. bld12 strains expressing the wildtype CrSAS- 6 tagged with HA, and bld10 strains expressing truncated Bld10p variants $(\Delta \mathrm{C} 2$ and $\Delta \mathrm{N} 3)$ have been described elsewhere ${ }^{8,21,31}$. $\mathrm{CrNN} 24 / \Delta \mathrm{C} 2$ and $\mathrm{CrNN} 24 / \Delta \mathrm{N} 3$ were produced using standard methods of genetic cross. Cells were grown in Tris-acetate-phosphate (TAP) medium ${ }^{51}$ with aeration on a $12 \mathrm{~h} / 12 \mathrm{~h}$ light/dark cycle, or under constant illumination with agitation.

Establishment of bld12 strains expressing full-length CrSAS-6 mutants. A genomic fragment containing the CrSAS-6 gene was cloned in pBluescript KS(+). N-domain mutations were introduced into the fragment by overlap extension PCR. The plasmids were digested with Bsp1407I, and 1.3-kb fragments containing the mutagenized sequences were subcloned in the original plasmid digested with Bsp1407I to replace the corresponding fragments. The sequences of the replaced region were verified by Sanger sequencing. Each plasmid was mixed with another plasmid, pSI103, carrying the selectable marker gene aphVIII (ref. 52), and introduced into bld 12 by electroporation ${ }^{53}$. The cells were cultivated on TAP agar plates containing $10 \mu \mathrm{g} \mathrm{ml}^{-1}$ paromomycin. For each CrSAS-6 mutant, about 500 colonies forming on the agar plates were isolated and subjected to western blot using anti-HA antibody. A clone expressing the CrSAS- 6 mutant most efficiently was used for further experiments.

Western blot of Chlamydomonas cells. Whole-cell extracts (10 $\mu \mathrm{g}$ per lane) were separated by SDS-PAGE and transferred to Immobilon-P membranes (Millipore). An affinity-purified rabbit polyclonal antibody against CrSAS-6 (ref. 8) (1:1,000), a rat monoclonal antibody against HA (clone $3 \mathrm{~F} 10$, Roche) $(1: 2,000)$, a rabbit antibody against the $120 \mathrm{~N}$-terminal residues of Bld10p (1:200), and a rabbit antibody against the C-terminal one-third of Bld10p (1:200) were used as primary antibodies $^{21,31}$. A horseradish-peroxidase-conjugated goat anti-rabbit-IgG (SigmaAldrich) (1:2,000) and a horseradish-peroxidase-conjugated goat anti-rat-IgG (Sigma-Aldrich) $(1: 3,000)$ were used as secondary antibodies.

Immunofluorescence microscopy of Chlamydomonas cells. Chlamydomonas reinhardtii cells grown in liquid medium were attached onto a glass slide coated with $0.1 \%$ polyethylenimine. The cells were fixed for $10 \mathrm{~min}$ in $-20^{\circ} \mathrm{C}$ methanol, washed three times in PBS, and incubated with a blocking solution $(10 \mathrm{mM}$ sodium phosphate buffer $\mathrm{pH} 7.2,5 \%$ normal goat serum, $5 \%$ glycerol, $1 \%$ fish gelatin, $0.004 \%$ sodium azide) for $30 \mathrm{~min}$ at $37^{\circ} \mathrm{C}$. The fixed cells were incubated for $1 \mathrm{~h}$ at $37^{\circ} \mathrm{C}$ with primary antibodies, rat monoclonal antibody against $\mathrm{HA}$ (clone $3 \mathrm{~F} 10$, Roche) and anti-acetylated $\alpha$-tubulin (monoclonal 6-11B-1, Sigma-Aldrich) both diluted to 1:100 in blocking solution, and washed three times for $15 \mathrm{~min}$ in PBS. The cells were stained for $1 \mathrm{~h}$ at $37^{\circ} \mathrm{C}$ with secondary antibodies, goat anti-rat IgG coupled to Alexa594 (Sigma-Aldrich) and goat anti-mouse IgG coupled to FITC (SigmaAldrich) diluted in blocking solution to 1:200, and washed three times for $15 \mathrm{~min}$ in PBS. Images were recorded using an Axioplan fluorescence microscope (Carl Zeiss MicroImaging) with a $63 \times 1.4$ NA plan-APOCHROMAT objective and a CoolSNAP CCD (charge-coupled device) camera (Roper Scientific).

Counting flagellated cells of Chlamydomonas. Log-phase cells were treated with gametic autolysin ${ }^{54}$ to remove the cell wall and induce flagellar growth. The cells were fixed with $1 \%$ glutaraldehyde and observed under a dark-field microscope. Percentages of flagellated cells were determined by counting cells with two, one and zero flagella.

Cloning, cell culture and transfection of human cells. Full-length HsSAS-6 NN24 (Q74E F131W H132R)-GFP was cloned in the Gateway compatible vector pEBTet-GW-EGFP (ref. 11). Briefly, the mutations were introduced by PCR in the pENTR-SD-Age-HsSAS-6 vector. After Sanger sequence verification, a Gateway reaction was performed to generate the final expression plasmid pEBTet-HsNN24-EGFP.

Human cells (AATC; not authenticated and not tested for mycoplasma) were grown in DMEM supplemented with GlutaMAX (Invitrogen), 10\% tetracyclinenegative fetal calf serum (GE Healthcare) and $1 \mu \mathrm{g} \mathrm{ml}^{-1}$ puromycin. To generate the inducible episomal iU2OS:HsNN24-GFP cell line, U2OS cells were transfected at $80 \%$ confluency in a $10 \mathrm{~cm}$ dish plate with the pEBTet-HsNN24-GFP construct using Lipofectamine 2000 (Life Technology). Transfected cells were selected with $1 \mu \mathrm{g} \mathrm{ml}^{-1}$ puromycin one day after transfection and amplified.

Early passage cells were used for the experiments, inducing expression with $1 \mu \mathrm{g} \mathrm{ml}^{-1}$ doxycycline for $48 \mathrm{~h}$ while depleting endogenous HsSAS-6 using an siRNA (Life Technology) targeting the $3^{\prime}$ UTR of HsSAS-6 as described previously ${ }^{11}$. Negative-control LO GC siRNA (Life Technology) was used as a negative control. Cells were fixed and stained $48 \mathrm{~h}$ after siRNA treatment.

Immunofluorescence microscopy for human cells. U2OS cells grown on glass coverslips were fixed for $7 \mathrm{~min}$ in $-20^{\circ} \mathrm{C}$ methanol, washed three times in PBS, then washed in PBST (PBS $+0.05 \%$ Tween- 20 ) and blocked in $1 \%$ bovine serum albumin in PBST for $30 \mathrm{~min}$. Cells were incubated for $4 \mathrm{~h}$ at room temperature with primary antibodies against GFP (rabbit 1:1,000, gift from V. Simanis, Swiss Federal Institute of Technology (EPFL), Lausanne, Switzerland) and centrin-2 (mouse 1:3,000, 20H5; gift from J. L. Salisbury, Mayo Clinic, USA), washed four times for $5 \mathrm{~min}$ in PBST, incubated for $45 \mathrm{~min}$ at room temperature with secondary antibodies (1:1,000, goat anti-rabbit coupled to Alexa488 and goat anti-mouse coupled to Alexa568 (Molecular Probes)), stained with $1 \mathrm{mg} \mathrm{ml}^{-1}$ Hoechst 33258, washed three times in PBST, and mounted (mounting medium: $4 \% n$-propyl-gallate; $1 \times$ PBS; $90 \%$ glycerol). For quantification of centriole number, only GFP-positive cells were considered for the analysis. Imaging was performed on a Zeiss LSM700 confocal microscope with a $63 \times / 1.4$ oil objective and a pinhole aperture corresponding to 1 airy unit. Optical sections were acquired every $0.31 \mu \mathrm{m}$, and planes containing centrioles were projected together. The resulting 16-bit images were processed using ImageJ.

A similar protocol was used for the immunofluorescence experiments presented in Fig. 5 and Supplementary Fig. 6 using the following antibodies: primary antibodies against either STIL (rabbit 1:1,000, Abcam, ab 89314) and GFP (mouse 1:100, Millipore, MAB3580), or GT335 (mouse 1:1,000, Adipogen, AG-20B-0020-C100), CP110 (rabbit 1:2,000, Proteintech, 12780-1-AP) and GFP-FITC (1:200, Abcam, ab 6662); secondary antibodies (1:1,000, either goat anti-rabbit coupled to Alexa568 and goat anti-mouse coupled to Alexa488, or else goat anti-mouse coupled to Cy5 and goat anti-rabbit coupled to Alexa568, all from Molecular Probes). For the acquisition of GT335 and CP110 images, optical sections were acquired on the LSM 700 every $0.13 \mu \mathrm{m}$ with a pixel size of $30 \mathrm{~nm}$. The distance between CP110 and GT335 was determined using ImageJ by performing a plot profile and measuring the distance between two maxima. Only centrioles in the proper orientation were analysed.

Stimulated emission depletion (STED) imaging and data processing. U2OS cells grown on glass coverslips $(18 \mathrm{~mm} \times 18 \mathrm{~mm}, 0.170 \pm 0.005 \mathrm{~mm}$, Zeiss $)$ were pre-extracted before fixation in BRB80 buffer ( $80 \mathrm{mM}$ K-PIPES, pH $6.8,1 \mathrm{mM}$ $\mathrm{MgCl}_{2}, 1 \mathrm{mM}$ EGTA) containing $0.5 \%$ Triton at $37^{\circ} \mathrm{C}$ for $30-60 \mathrm{~s}$. Cells were then fixed for $10 \mathrm{~min}$ in $4 \%$ paraformaldehyde at room temperature, washed in PBS, then incubated for $1 \mathrm{~h}$ at room temperature in $1 \%$ bovine serum albumin in PBST with primary antibodies against Cep164 (rabbit 1:2,000, Novus Biologicals) or Cep63 (rabbit 1:1,000, Millipore) and GFP (mouse 1:100, Millipore), washed twice for $5 \mathrm{~min}$ in PBST, incubated for $1 \mathrm{~h}$ at room temperature with secondary antibodies $(1: 1,000$, goat anti-rabbit coupled to Alexa488 and goat anti-mouse coupled to Alexa568 (Molecular Probes)), stained with $1 \mathrm{mg} \mathrm{ml}^{-1}$ Hoechst 33258, washed once in PBST, and mounted. Imaging of GFP-expressing cells was performed on a Leica SP5WL+STED confocal microscope with a $100 \times$ objective (APO $100 \times 1.4$ oil). Images were acquired using the following parameters: $60 \%$ laser, gating of $0.5-6.5$, accumulation of 4 , zoom 4 , speed of $200 \mathrm{~Hz}$, format $2,048 \times 2,048$. The resulting 12-bit images were processed using ImageJ.

Accession numbers. Structural coordinates have been deposited at the Protein Data Bank (PDB) under accession numbers 4TPZ (CrNN2), 4TQ7 (CrNN10), 4TTW (CrNN18), 4TTX (CrNN19), 4U2I (CrNN23), 4TO7 (CrNN24), 4TTY (CrNN25), $4 \mathrm{TTZ}(\mathrm{CrNN} 26)$ and 4U2J (CrNN27).

35. Olieric, N. et al. Automated seamless DNA co-transformation cloning with direct expression vectors applying positive or negative insert selection. BMC Biotechnol. 10, 56 (2010). 
36. Laue, T.M. et al. In Analytical Ultracentrifugation in Biochemistry and Polymer Science 90-125 (The Royal Society of Chemistry, 1992).

37. Humphrey, W., Dalke, A. \& Schulten, K. VMD: visual molecular dynamics. J. Mol. Graph. 14, 33-38 (1996).

38. Case, D. A. et al. The Amber biomolecular simulation programs. J. Comput. Chem. 26, 1668-1688 (2005)

39. Phillips, J. C. et al. Scalable molecular dynamics with NAMD. J. Comput. Chem. 26, 1781-1802 (2005).

40. MacKerell, A. D. Jr, Banavali, N. \& Foloppe, N. Development and current status of the CHARMM force field for nucleic acids. Biopolymers 56, 257-265 (2000).

41. Hilbert, M. et al. Caenorhabditis elegans centriolar protein SAS- 6 forms a spiral that is consistent with imparting a ninefold symmetry. Proc. Natl Acad. Sci. USA 110, 11373-11378 (2013).

42. Wright, R. L., Salisbury, J. \& Jarvik, J. W. A nucleus-basal body connector in Chlamydomonas reinhardtii that may function in basal body localization or segregation. J. Cell Biol. 101, 1903-1912 (1985).

43. Bornens, M., Paintrand, M., Berges, J., Marty, M. C. \& Karsenti, E. Structural and chemical characterization of isolated centrosomes. Cell Motil. Cytoskeleton 8, 238-249 (1987).

44. Heymann, J. B. \& Belnap, D. M. Bsoft: image processing and molecular modeling for electron microscopy. J. Struct. Biol. 157, 3-18 (2007).
45. Frank, J. et al. SPIDER and WEB: processing and visualization of images in 3D electron microscopy and related fields. J. Struct. Biol. 116, 190-199 (1996).

46. Kabsch, W. XDS. Acta Crystallogr. D 66, 125-132 (2010).

47. McCoy, A. J. et al. Phaser crystallographic software. J. Appl. Crystallogr. 40, 658-674 (2007).

48. Emsley, P. \& Cowtan, K. Coot: model-building tools for molecular graphics. Acta Crystallogr. D 60, 2126-2132 (2004).

49. Adams, P. D. et al. PHENIX: building new software for automated crystallographic structure determination. Acta Crystallogr. D 58, 1948-1954 (2002).

50. Biasini, M. et al. SWISS-MODEL: modelling protein tertiary and quaternary structure using evolutionary information. Nucleic Acids Res. 42, W252-W258 (2014).

51. Gorman, D. S. \& Levine, R. P. Cytochrome $f$ and plastocyanin: their sequence in the photosynthetic electron transport chain of Chlamydomonas reinhardi. Proc. Nat Acad. Sci. USA 54, 1665-1669 (1965).

52. Sizova, I., Fuhrmann, M. \& Hegemann, P. A Streptomyces rimosus aphVIII gene coding for a new type phosphotransferase provides stable antibiotic resistance to Chlamydomonas reinhardtii. Gene 277, 221-229 (2001).

53. Shimogawara, K., Fujiwara, S., Grossman, A. \& Usuda, H. High-efficiency transformation of Chlamydomonas reinhardtii by electroporation. Genetics 148 , 1821-1828 (1998).

54. Harris, E. H. The Chlamydomonas Sourcebook (Academic, 1989) 


\title{
Corrigendum: SAS-6 engineering reveals interdependence between cartwheel and microtubules in determining centriole architecture
}

\author{
Manuel Hilbert, Akira Noga, Daniel Frey, Virginie Hamel, Paul Guichard, Sebastian H. W. Kraatz, Moritz \\ Pfreundschuh, Sarah Hosner, Isabelle Flückiger, Rolf Jaussi, Mara M. Wieser, Katherine M. Thieltges, Xavier \\ Deupi, Daniel J. Müller, Richard A. Kammerer, Pierre Gönczy, Masafumi Hirono and Michel O. Steinmetz
}

Nature Cell Biology 18, 393-403 (2016); published online 21 March 2016; corrected after print 28 April 2016

In the version of this Article originally published, Paul Guichard should not have been listed under the statement 'These authors contributed equally to this work? This has been corrected in all online versions of the Article. 
a
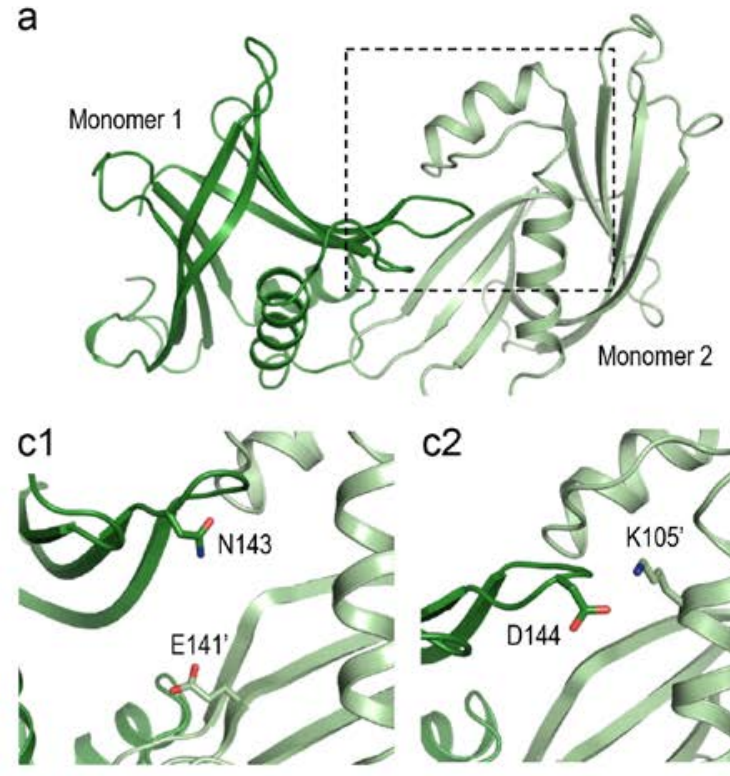

c2

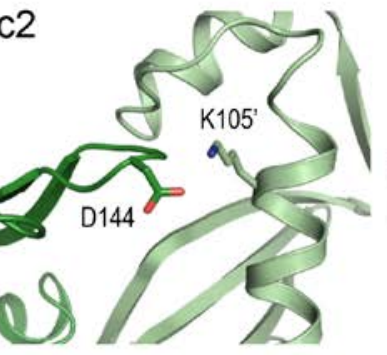

c4
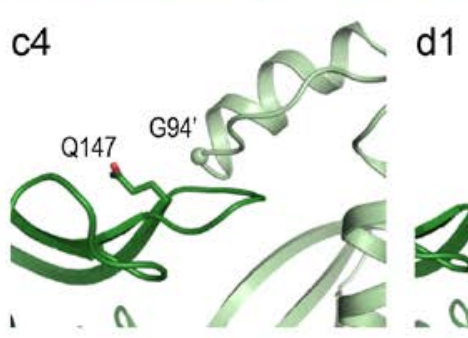

e

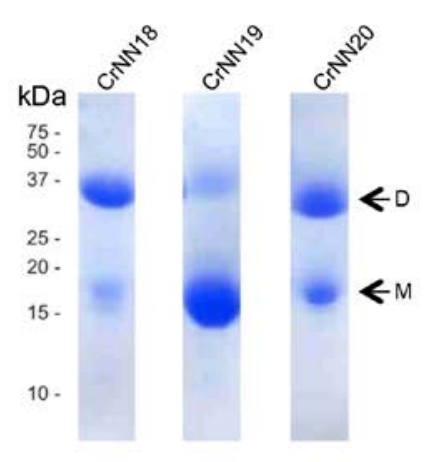

f b

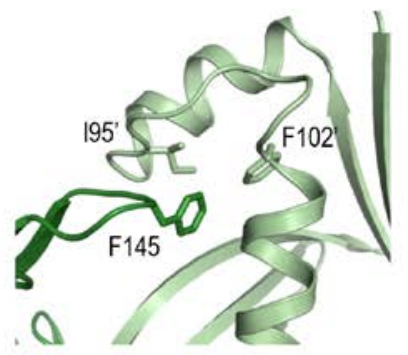

c3

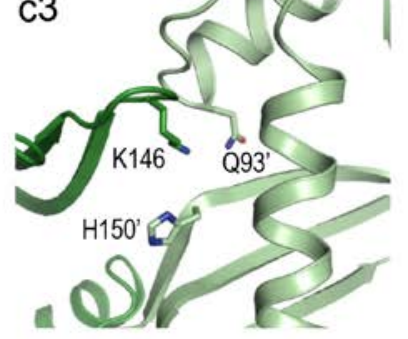

d2
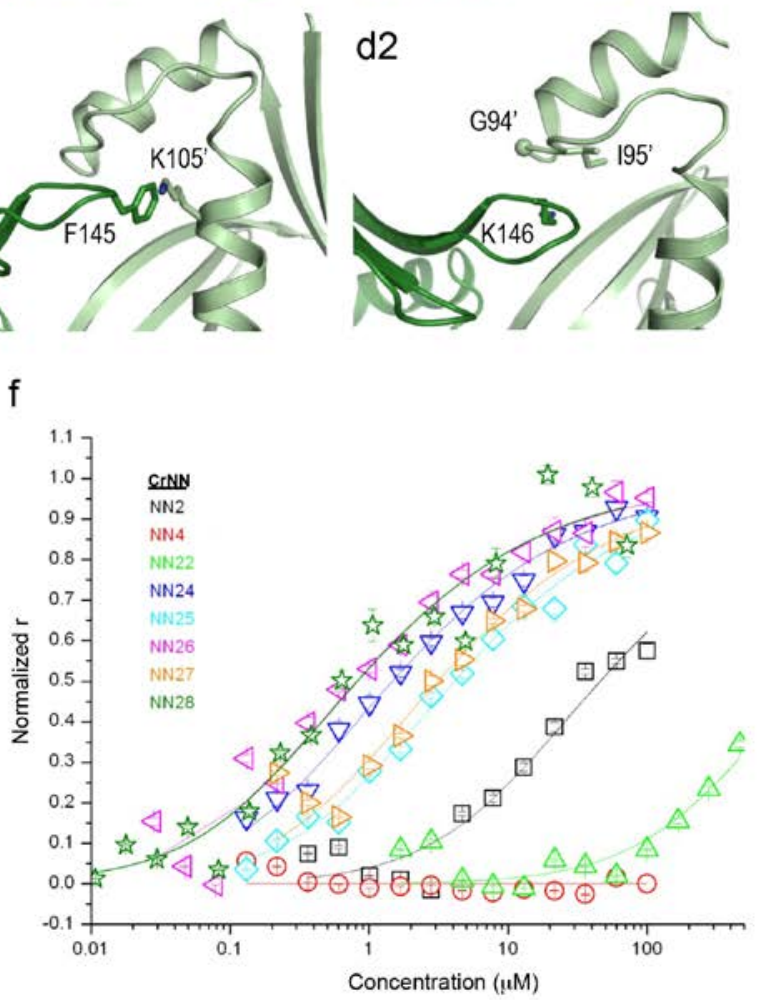

Supplementary Figure 1 Mutagenesis of the CrSAS- 6 N-N dimer interface. (a) Overall view of the crystal structure of the wild type CrSAS-6 N-N dimer (PDB ID 3Q0X). Monomers 1 and 2 of the dimer are shown as cartoon representations and are colored in dark and light green, respectively. The dashed box highlights the excerpt shown in panels (b) to (d). (b-d) Close-up views of the CrSAS- $6 \mathrm{~N}-\mathrm{N}$ dimer interface showing in stick representation the residues that were targeted for the 'hydrophobic contact' (b), 'salt bridge' (c) and 'covalent linkage' (d) mutagenesis approaches. See Supplementary Table 1 for a full list of the mutants that were analyzed in the course of this study. (e) SDS-PAGE analysis of the CrSAS-6 N-N dimer mutants that were generated based on the 'covalent linkage' mutagenesis approach. Nonreducing SDS-PAGE of variants in which pairs of cysteine residues were introduced at positions 105 and 145 (CrNN18), 94 and 146 (CrNN19), and 95 and 146 (CrNN20) to allow the formation of covalently linked N-N dimers under oxidizing conditions. Samples were air-oxidized overnight prior to SDS-PAGE analysis. M, migration position for the monomers; D, migration position for the disulfide-bridge linked dimers. (f) Stabilities of selected CrSAS- 6 N-N dimer variants determined by FP dilution experiments. See also Table 1. Each data point represents the average from five independent measurements. $r$, anisotropy value. 


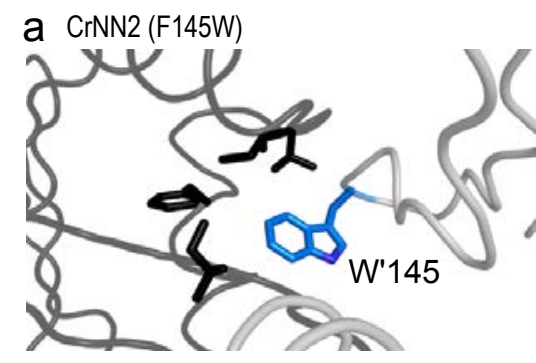

b CrNN10 (Q93E)

C CrNN18 (K105C, F145C)

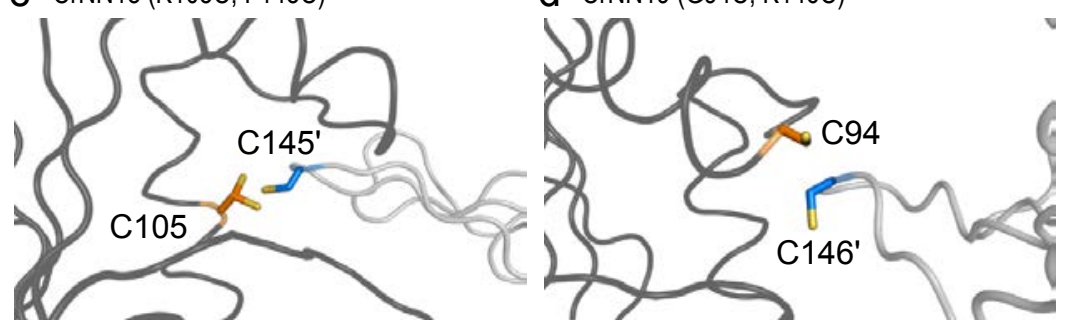

e CrNN23 (Q93E, G94D, K146R, Q147R) f CrNN24 (Q93E, F145W, K146R)
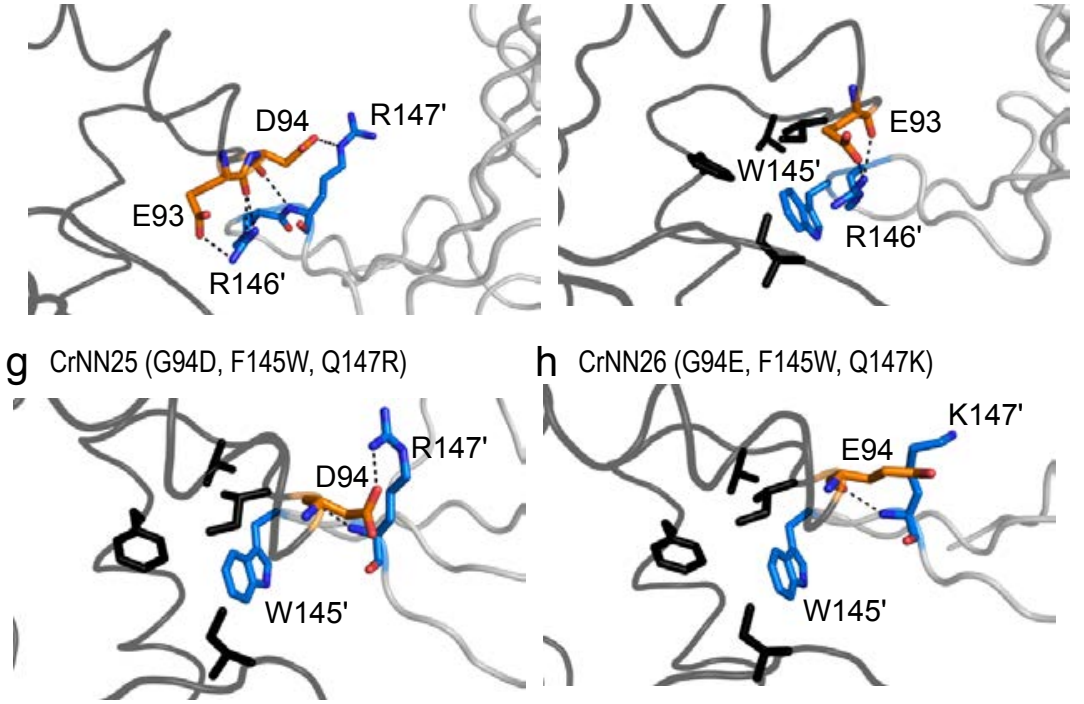

i CrNN27 (Q93E, F145W)

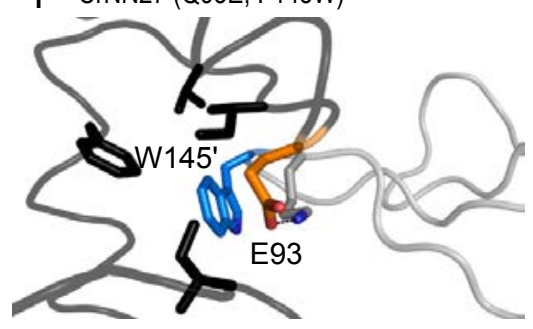

Supplementary Figure 2 Crystal structures of CrSAS- 6 N-N dimer variants. Close-up views of the dimer interfaces of mutant CrSAS- 6 N-N dimers solved by X-ray crystallography (Supplementary Table 2). Mutated residues are shown in sticks representation and are colored in orange (monomer 1) and blue (monomer 2). Residues in the vicinity of the mutated residues are shown in black (monomer 1 ) or gray (monomer 2 ). 


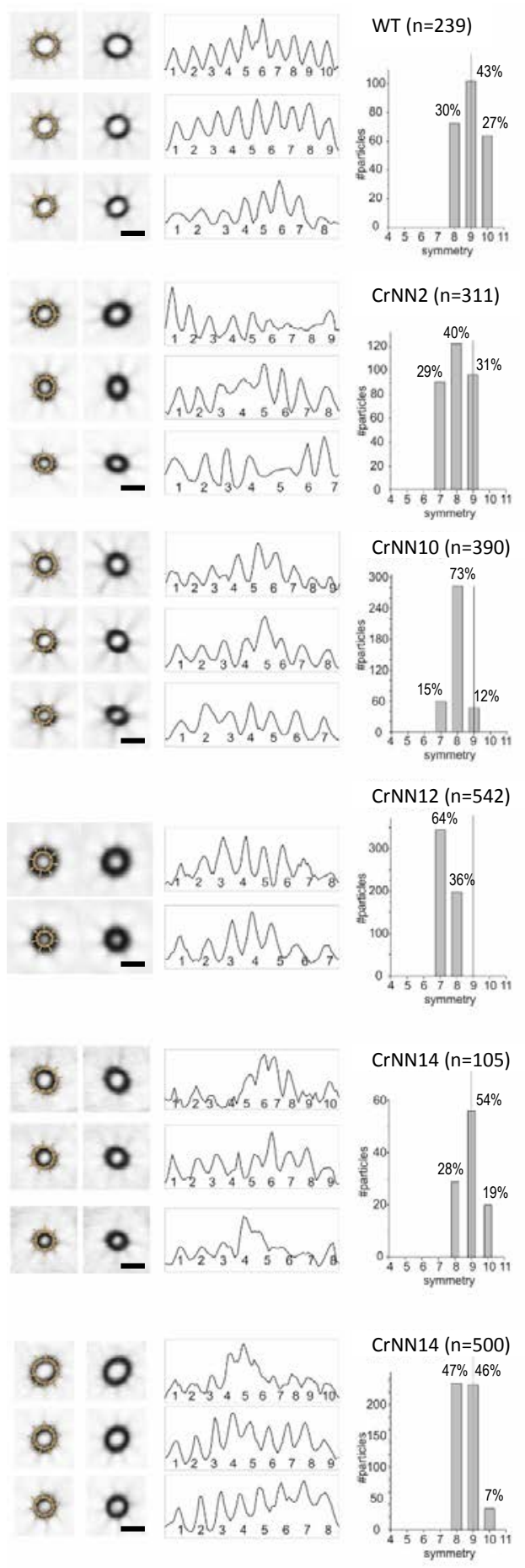

Supplementary Figure 3 Symmetry analysis of CrSAS- 6 N-CC cartwheels assembled in vitro. The symmetries of all CrSAS- 6 cartwheel variants generated in this study were assessed either by AFM or EM and subsequent single particle image analysis. Wild type (WT), CrNN2, CrNN10, CrNN12, CrNN14 and CrNN15 were analyzed by AFM (panels on the left side); CrNN20, CrNN23, CrNN24, CrNN25, CrNN26, CrNN27 and CrNN28 were

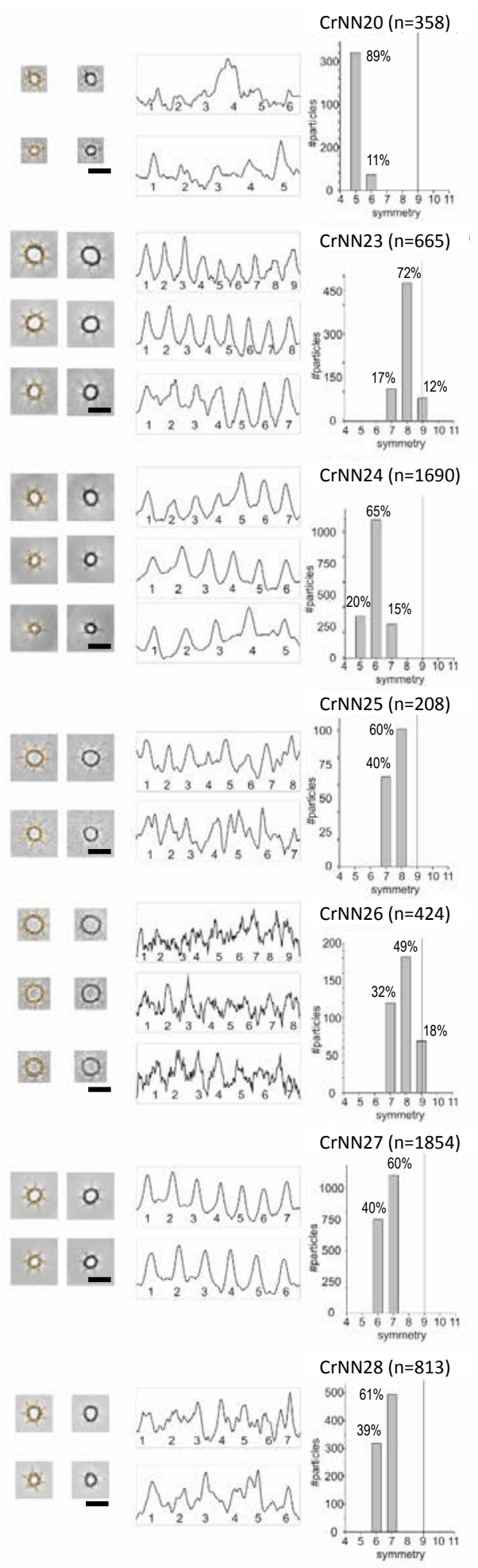

analyzed by negative stain EM (panels on the right side). See also Table 1. Scale bars, $25 \mathrm{~nm}$. $\mathrm{n}$, number of cartwheel specimens analyzed. WT, CrNN2, CrNN10, CrNN12, CrNN14, CrNN15, CrNN23, CrNN24, CrNN25 and $\mathrm{CrNN} 27$ were analyzed in the CrSAS-6 N-CC context (residues 1-503); CrNN20, CrNN26 and CrNN28 were analyzed in the CrSAS-6 6HR context (residues 1-226). 
a

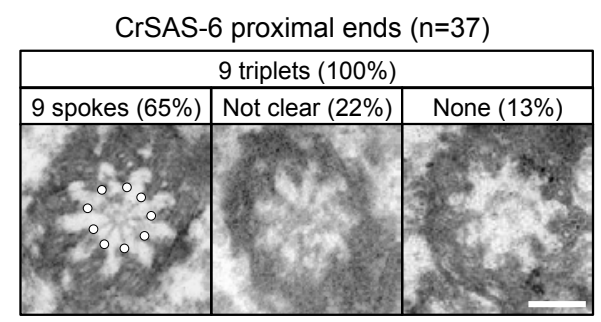

b

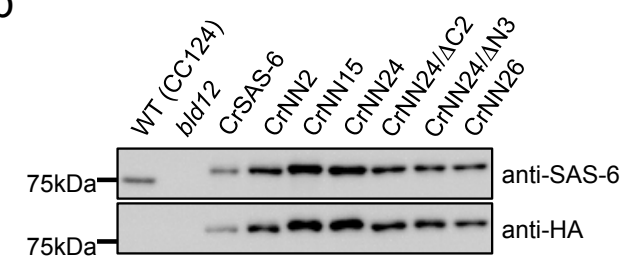

d

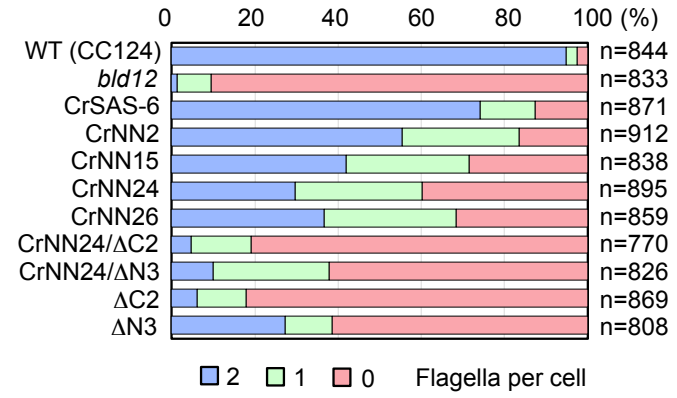

e

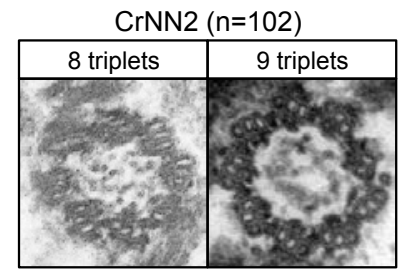

$f$

CrNN15 $(n=106)$
C
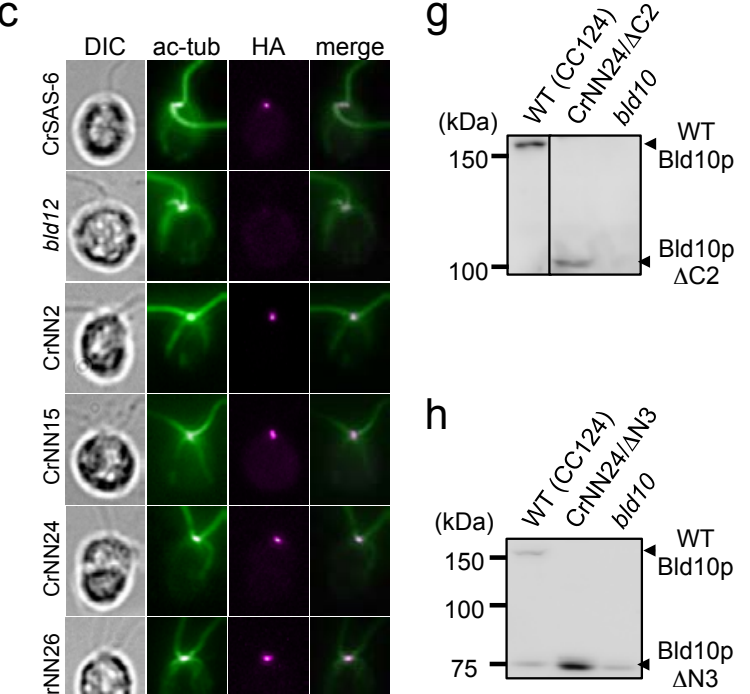
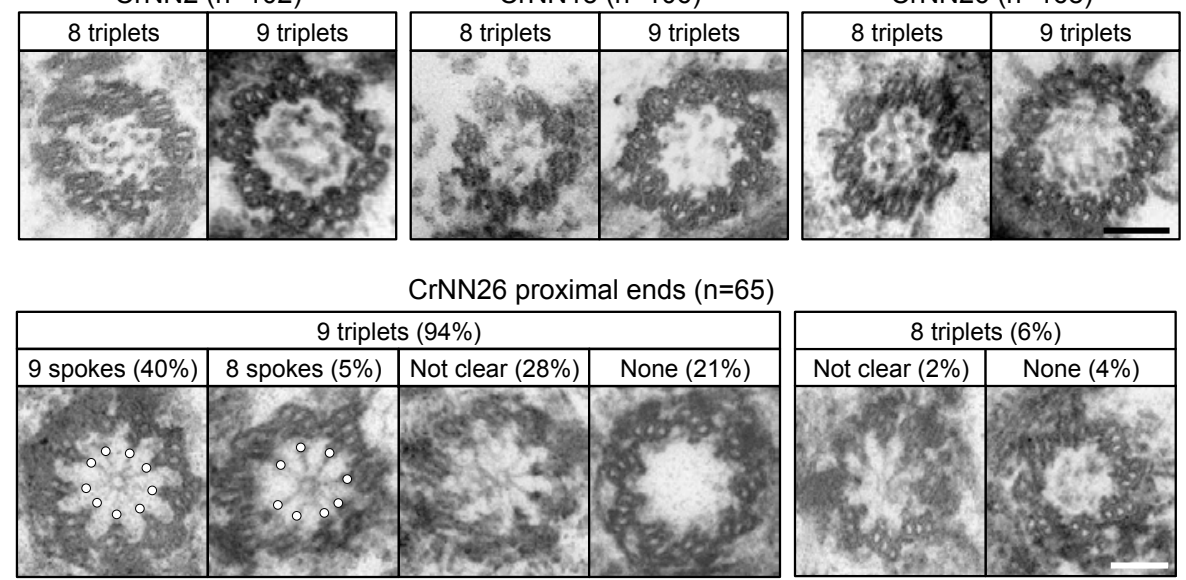

Supplementary Figure 4 Establishment of Chlamydomonas strains. (a) Cross section electron micrographs of the proximal ends of CrSAS- 6 centrioles. Images were classified into three groups: those containing cartwheels with 9 spokes, cartwheels with an unclear number of spokes (Not clear), and no visible cartwheels (None). Percentages of centrioles in these three classes are indicated in parentheses. Spokes are highlighted with white dots. (b) Westernblot analyses of whole-cell lysates of cells expressing wild type (CC124), bld12, CrSAS-6, CrNN2, CrNN15, CrNN24, CrNN24/AC2, CrNN24/AN3, and $\mathrm{CrNN} 26$ using antibodies against CrSAS-6 (upper blots) and HA (lower blot). The apparent molecular weight of CrSAS- 6 variants is slightly larger than that of endogenous CrSAS- 6 because of the hemagglutinin (HA) tag fused at its C-terminus. (c) Indirect immunofluorescence of CrSAS-6, bld12, CrNN2, CrNN15, CrNN24, CrNN26, CrNN24/AC2, and CrNN24/AN3 expressing cells using antibodies against acetylated $\alpha$-tubulin (ac-tub, green) and HA (magenta). Left-most panels show differential interference images (DIC). At least 16 cells were observed for each strain, and representative images are shown. Scale bar, $5 \mu \mathrm{m}$. (d) Percentages of flagellated CC124, b/d12, CrSAS-6, CrNN2, CrNN15, CrNN24, CrNN26, CrNN24/AC2, CrNN24/AN3, $\Delta \mathrm{C} 2$, and $\Delta \mathrm{N} 3$ expressing cells. Cells with two (blue), one (green), and zero (pink) flagella were counted. $n$, number of cells counted. (e) Cross section electron micrographs of CrNN2, CrNN15, and CrNN26 centrioles. (f) Cross section electron micrographs of the proximal ends of CrNN26 centrioles. Images of 9- and 8-triplet centrioles were classified into four groups as in Fig. 3c. Percentages of centrioles in these classes are indicated in parentheses. Spokes are highlighted with white dots. Scale bars (a, e, f), $100 \mathrm{~nm} .(\mathrm{g}, \mathbf{h})$ Western-blot analyses of whole-cell lysate of CC124, CrNN24/AC2, CrNN24/ $\Delta \mathrm{N} 3$, and b/d10 expressing cells using antibodies against the $\mathrm{N}$-terminal 120 residues of Bld10p (g) or the C-terminal one-third of Bld10p (h). The bands at $75 \mathrm{kDa}$ that appear in the lanes of CC124 and b/d10 in the right panel are non-specific, and the band of $\Delta \mathrm{N} 3$ overlaps with the non-specific band. Each western-blot analysis (panels $b, g$ and $h$ ) was performed once; the unprocessed, original scans are shown in Supplementary Fig. 7. 
a

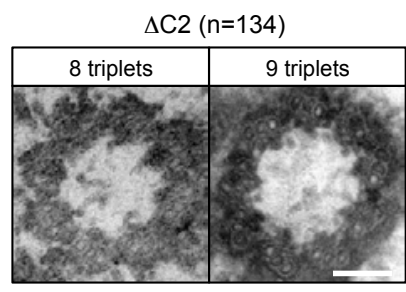

C

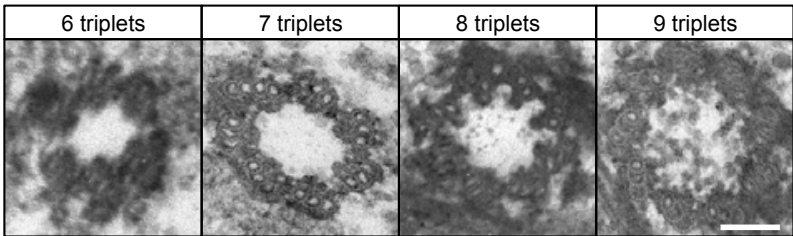

$\Delta \mathrm{C} 2$ proximal ends $(n=40)$

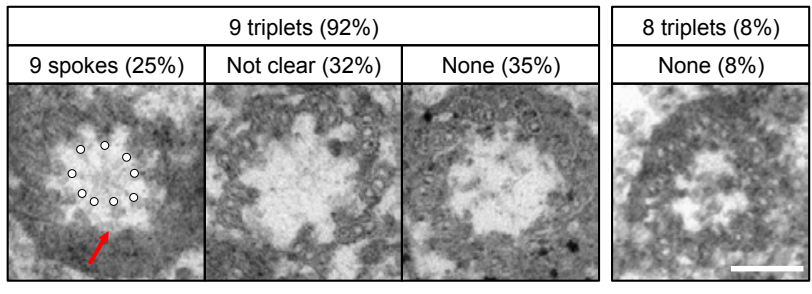

d

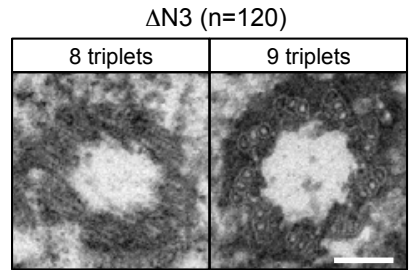

e

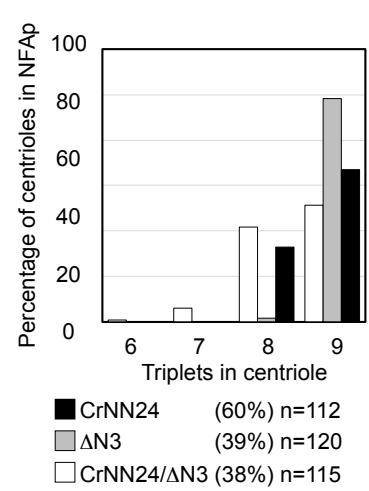

9

CrNN24/AN3 proximal ends $(n=76)$

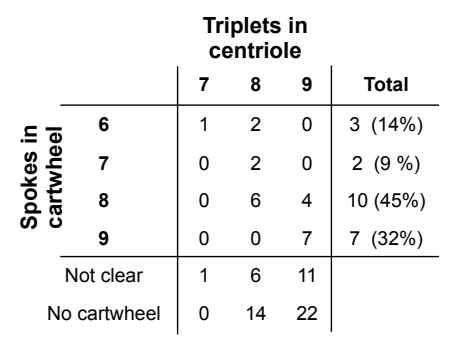

f

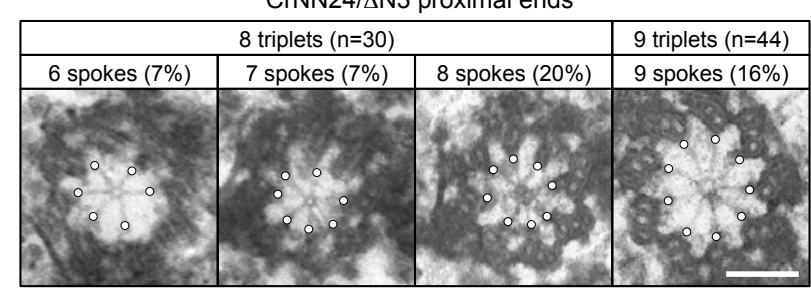

h

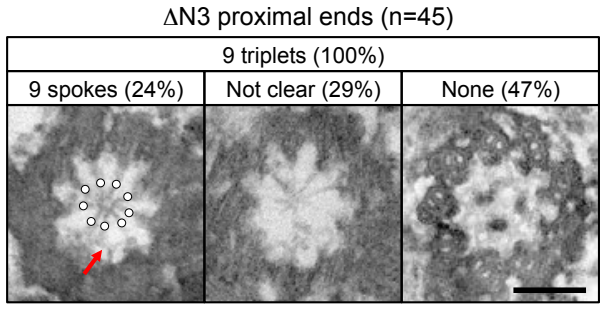

Supplementary Figure 5 Centrioles and cartwheels in Chlamydomonas strains. (a) Cross section electron micrographs of $\Delta \mathrm{C} 2$ centrioles. (b) Cross section electron micrographs of the proximal ends of $\Delta \mathrm{C} 2$ centrioles. Images of 9- and 8-triplet centrioles were classified into three groups as in Supplementary Fig. 4a. Percentages of centrioles in these classes are indicated in parentheses. Spokes are highlighted with white dots. Cartwheel spokes are often detached from triplet microtubules as indicated by a red arrow. (c) Cross section electron micrographs of CrNN24/ $\Delta$ N3 centrioles. (d) Cross section electron micrographs of $\Delta \mathrm{N} 3$ centrioles. (e) Triplet number distributions of centrioles in NFAp preparations from CrNN24 (black), $\Delta$ N3 (gray), and CrNN24/AN3 (white) expressing cells. Percentages of flagellated cells in these strains (Supplementary Fig. 4d) are indicated in parentheses. $\mathrm{n}$, number of centrioles observed. (f) Cross section electron micrographs of the proximal ends of CrNN24/AN3 centrioles. Spokes are highlighted with white dots. (g) Distributions of triplet and spoke numbers of CrNN24/ $\Delta \mathrm{N} 3$ centrioles. 76 electron micrographs of centriole cross sections of the proximal ends of the $\mathrm{CrNN} 24 / \Delta \mathrm{N} 3$ strain were classified into 18 groups based on their numbers of triplets and cartwheel spokes, and appearance of the cartwheel. Numbers and percentages of the micrographs in these groups are listed. (h) Cross section electron micrographs of the proximal ends of $\Delta N 3$ centrioles. Images of 9-triplet centrioles were classified into three groups as in Supplementary Fig. 4a. Percentages of centrioles in these classes are indicated in parentheses. Spokes are highlighted with white dots. Cartwheel spokes are often detached from triplet microtubules as indicated by a red arrow. Scale bars, $100 \mathrm{~nm}$. The CrSAS- $6 \mathrm{~N}$-domain mutations for all experiments were introduced in the full length protein. 


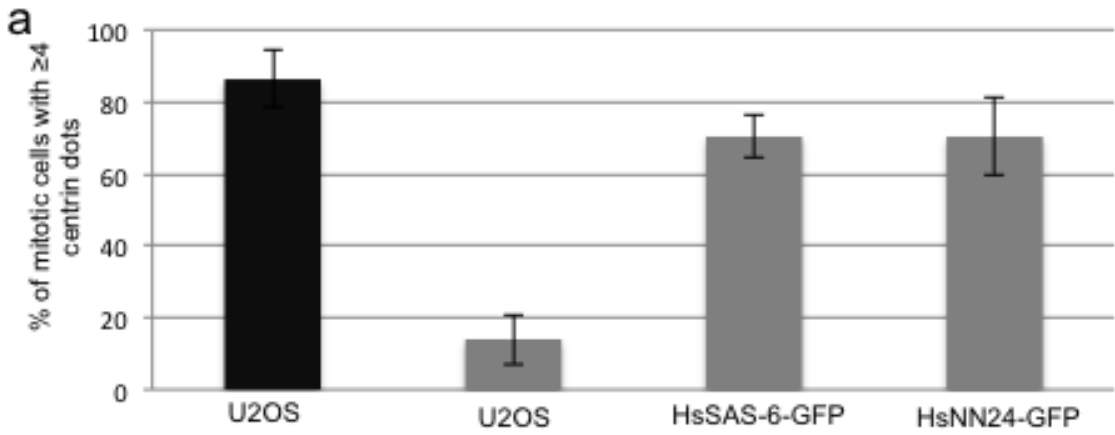

\section{U2OS}
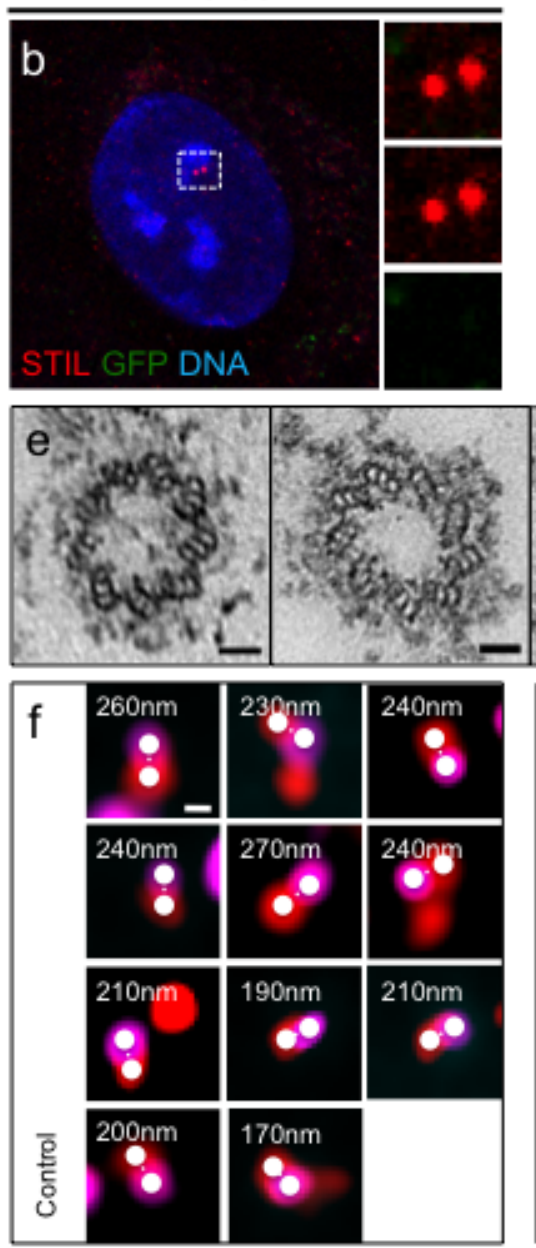

HsSAS-6-GFP siRNA HsSAS-6 3'UTR

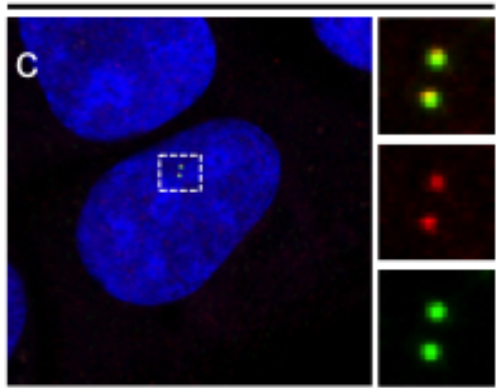

HsNN24-GFP SIRNA HsSAS-6 3 'UTR

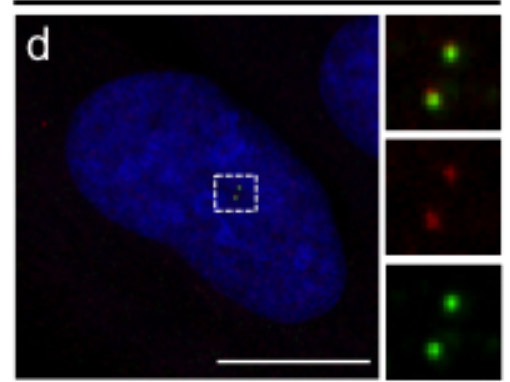

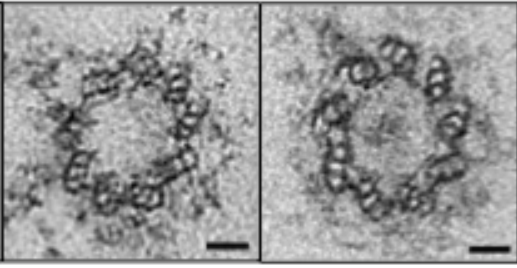
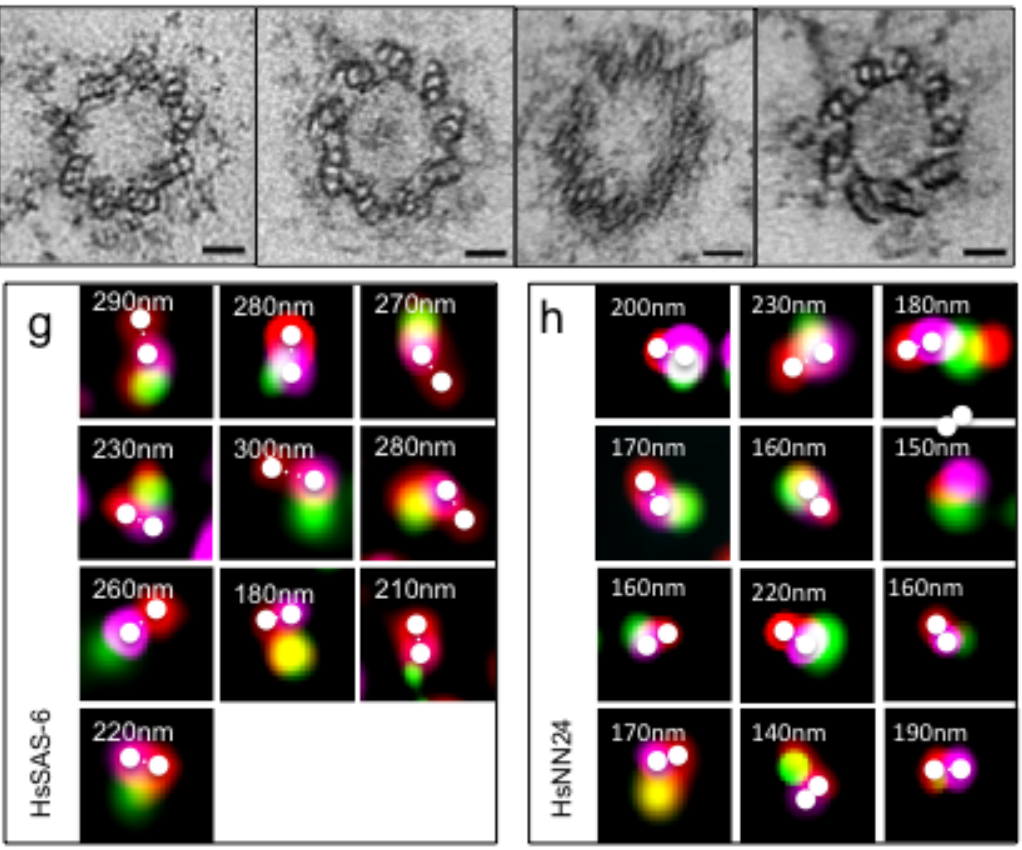

Supplementary Figure 6 Expression and localization of HsSAS-6 NN24 in human cells depleted of endogenous HsSAS-6. (a) Quantification of experiments shown in Figure 5 (b-e), average $+/-S D$ and $n$ (number of centrioles analyzed) were as follows: U20S, control 86.5 +/- 8, 229; U2OS + HsSAS-6 siRNA, 14 +/- 7, 282; HsSAS-6-GFP + HsSAS-6 siRNA, 70.5 +/- 6, 301; HsNN24-GFP + HsSAS-6 siRNA, 70.5 +/- 11, 118. Data from at least two independent experiments (50 cells/experiment; error bar indicates $\mathrm{SD}$ ). Black columns correspond to control whereas grey columns correspond to siRNAs targeting HsSAS-6. (b-d) Interphase control U2OS cells (b), as well as iU2OS:HsSAS-6-GFP (c) or iU2OS:HsNN24-GFP (d) cells depleted of endogenous HsSAS-6 induced concomitantly with doxycycline, fixed after $48 \mathrm{hr}$, and stained with antibodies against STIL (red) and GFP (green); DNA in blue. Insets show magnified view of the delineated centrosome regions. Scale bar, $10 \mu \mathrm{m}$. Representative images from two independent experiments. (e) Cross-section EM images of centrioles from cells expressing HsNN24-GFP and depleted from endogenous HsSAS-6. Note that all 6 centrioles shown here are 9-fold symmetrical. Scale bar, $50 \mathrm{~nm}$. Data from two independent experiments. (f-h) Centrioles from control U20S cells (f), as well as iU2OS: HsSAS-6-GFP (g) or iU2OS:HsNN24-GFP (h) cells depleted of endogenous HsSAS- 6 and induced concomitantly with doxycycline (g, h), fixed after $48 \mathrm{hr}$, and stained with antibodies against CP110 (red), GT335 (magenta) and GFP-FITC (green). Note that only insets of centrioles are shown, with the distance between CP110 and GT335 signals indicated in each case. Scale bar, $200 \mathrm{~nm}$. Data from one experiment. The HsSAS-6 $\mathrm{N}$-domain mutations for all experiments were introduced in the full length protein. Source data for a is available in Supplementary Table 4. 
Supplementary Fig. 4b

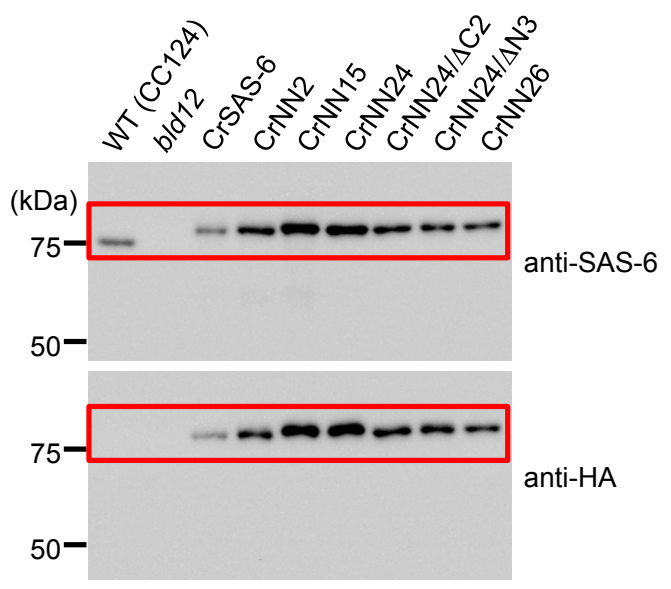

Supplementary Fig. 4g

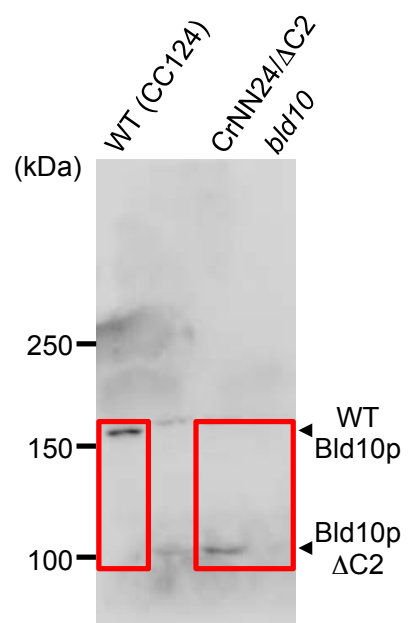

Supplementary Fig. 4h

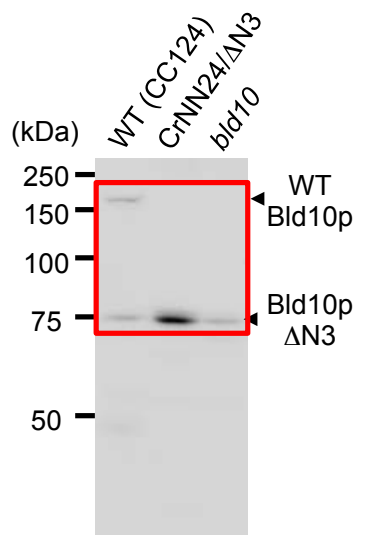

Supplementary Figure 7 Uncropped scans of western blots produced in this study. The cropped regions shown in Supplementary Fig. 4bgh are indicated by red boxes. 


\begin{tabular}{llll}
$\begin{array}{lll}\text { CrSAS-6 N-N } \\
\text { variant }\end{array}$ & Mutations & $\mathrm{K}_{\mathrm{D} \text { AUC }}$ & $\mathrm{K}_{\mathrm{D} \text { ITC/FP }}$ \\
& $\mu \mathrm{M}$ & $\mu \mathrm{M}$ \\
\hline WT & - & $350 \pm 30$ & $76 \pm 4^{\mathrm{a}}$
\end{tabular}

\section{Hydrophobic contact}

$\begin{array}{ll}\text { CrNN1 } & \text { F145M } \\ \text { CrNN2 } & \text { F145W } \\ \text { CrNN3 } & 195 \mathrm{~F} \\ \text { CrNN4 } & \text { 195V F102W } \\ \text { CrNN5 } & \text { F102W F145Y } \\ \text { CrNN21 } & 195 \mathrm{~V}\end{array}$

$3000 \pm 200$ n.d.

$78 \pm 4 \quad 30 \pm 10^{\mathrm{a}}$

$3000 \pm 300 \quad 4000 \pm 6000^{a}$

$20000 \pm 200 \quad 6000 \pm 19000^{\mathrm{a}}$

$3400 \pm 200 \quad 1700 \pm 200^{a}$

$8000 \pm 4000$ n.d.

\section{Salt bridge}

$\begin{array}{ll}\text { CrNN6 } & \text { K105R } \\ \text { CrNN7 } & \text { K105R D144E } \\ \text { CrNN8 } & \text { N143R } \\ \text { CrNN10 } & \text { Q93E } \\ \text { CrNN12 } & \text { Q93E K146R } \\ \text { CrNN13 } & \text { G94D Q147K } \\ \text { CrNN14 } & \text { G94E Q147K } \\ \text { CrNN15 } & \text { G94D Q147R } \\ \text { CrNN16 } & \text { G94E Q147R } \\ \text { CrNN17 } & \text { H150R }\end{array}$

$\begin{array}{ll}1330 \pm 70 & 620 \pm 60^{\mathrm{a}} \\ 2730 \pm 20 & 3000 \pm 3000^{\mathrm{a}} \\ \text { n.d. } & 300 \pm 150^{\mathrm{a}} \\ 136 \pm 4 & 33 \pm 4^{\mathrm{a}} \\ 58 \pm 2 & 14 \pm 1^{\mathrm{a}} \\ 20 \pm 10 & 55 \pm 8^{\mathrm{a}} \\ 21 \pm 0.3 & 20 \pm 10^{\mathrm{a}} \\ 20.3 \pm 0.1 & 23 \pm 7^{\mathrm{a}} \\ 22.5 \pm 0.3 & 270 \pm 90^{\mathrm{a}} \\ 2200 \pm 300 & \text { n.d. }\end{array}$

\section{Covalent linkage ${ }^{c}$}

$\begin{array}{llll}\text { CrNN18 } & \text { K105C F145C } & \text { n.d. } & \text { n.d. } \\ \text { CrNN19 } & \text { G94C K146C } & \text { n.d. } & 510 \pm 90^{a} \\ \text { CrNN20 } & \text { I95C K146C } & 600 & \text { n.d. }\end{array}$

\section{Combination of hydrophobic contact and salt bridge}

\begin{tabular}{llll} 
CrNN23 & Q93E G94D K146R Q147R & $1 \pm 0.5$ & n.d. \\
CrNN24 & Q93E F145W K146R & $2.3 \pm 0.1$ & $0.74 \pm 0.04^{\mathrm{b}}$ \\
CrNN25 & G94D F145W Q147R & $1.6 \pm 0.1$ & $2 \pm 0.1^{\mathrm{b}}$ \\
CrNN26 & G94E F145W Q147K & $4 \pm 3$ & $0.4 \pm 0.05^{\mathrm{b}}$ \\
CrNN27 & Q93E F145W & $9 \pm 7$ & $1.6 \pm 0.2^{\mathrm{b}}$ \\
CrNN28 & Q93E G94D F145W K146R Q147K & $0.5 \pm 0.3$ & $0.4 \pm 0.2^{\mathrm{b}}$ \\
\hline
\end{tabular}

${ }^{\mathrm{a}} \mathrm{K}_{\mathrm{D}}$ determined by ITC experiment.

${ }^{\mathrm{b}} \mathrm{K}_{\mathrm{D}}$ determined by FP experiments.

${ }^{c} K_{D}$ determined under reducing buffer conditions

n.d., not determined.

The table is divided into blocks to highlight: WT, wild type; CrNN1-CrNN5 and CrNN21, the 'hydrophobic contact' approach; CrNN6-CrNN17, the 'salt bridge' approach; CrNN18-CrNN20, the 'covalent linkage' approach; and CrNN23-CrNN28, combinations of CrNN1-CrNN17. See Text and Supplementary Fig. 1 for details. +/- indicate SD. 


\begin{tabular}{|c|c|c|c|c|c|c|c|c|c|}
\hline & CrNN2 & CrNN10 & CrNN18 & CrNN19 & CrNN23 & CrNN24 & CrNN25 & CrNN26 & CrNN27 \\
\hline \multicolumn{10}{|l|}{ Data collection } \\
\hline Space group & P212121 & P212121 & C121 & P1211 & P 212121 & C2221 & C121 & P212121 & P212121 \\
\hline \multicolumn{10}{|l|}{ Cell dimensions } \\
\hline$a, b, c(\AA)$ & $40.7,97.6,143.2$ & $46.1,73.6,95.0$ & $98.0,29,3,63.7$ & $87.6,95.6,91.4$ & $40.2,96.6,142.5$ & $42.5,67.4,104.2$ & $117.2,44.2,70.3$ & $96.5,40.1,142.5$ & $40.2,97.0,142.8$ \\
\hline$\alpha, \beta, \gamma(\Upsilon)$ & $90,90,90$ & $90,90,90$ & $90,127,90$ & $90,112.6,90$ & $90,90,90$ & $90,90,90$ & $90,117.4,90$ & $90,90,90$ & $90,90,90$ \\
\hline Resolution (Å) & $57.72-1.8(1.85-1.80)$ & $41.44-2.64(2.71-2.64)$ & $50.9-1.2(1.23-1.20)$ & $84.4-2.5(2.56-2.50)$ & $48.32-2.28(2.34-2.28)$ & $52.09-1.25(1.28-1.25)$ & $40.7-1.9(1.95-1.90)$ & $71.25-2.50(2.56-2.50)$ & $80.24-2.00(2.05-2.00)$ \\
\hline$R_{\text {merge }}$ & $0.063(0.926)$ & $0.089(0.514)$ & $0.039(1.388)$ & $0.224(2.017)$ & $0.083(0.59)$ & $0.046(5.617)$ & $0.120(1.390)$ & $0.115(1.123)$ & $0.117(1.029)$ \\
\hline$|/ \sigma|$ & $22.63(2.21)$ & $25.06(6.26)$ & $16.20(1.35)$ & $6.78(0.72)$ & $21.46(3.47)$ & $17.42(0.44)$ & $14.83(1.66)$ & $23.94(2.66)$ & $15.66(2.14)$ \\
\hline Completeness (\%) & 99.9 (98.9) & $99.7(97.1)$ & 99.0 (95.5) & $99.6(97.7)$ & 99.9 (73.2) & $98.7(84.0)$ & $98.4(93.8)$ & 99.1 (87.9) & $100(100)$ \\
\hline Redundancy & $7.3(6.7)$ & 12.5 (11.9) & $6.4(6.1)$ & $3.8(3.6)$ & $9.8(6.9)$ & $12.4(10.1)$ & $7.5(7.1)$ & $14.1(11.4)$ & $7.3(7.4)$ \\
\hline \multicolumn{10}{|l|}{ Refinement } \\
\hline Resolution (Å) & $57.72-1.8$ & $41.44-2.65$ & $20.78-1.20$ & $63.26-2.50$ & $48.32-2.28$ & $19.66-1.25$ & $40.70-1.90$ & $71.25-2.50$ & $80.24-2.0$ \\
\hline No. reflections & 53812 & 9912 & 45554 & 48225 & 25555 & 41116 & 25239 & 19736 & 38651 \\
\hline$R_{\text {work }} / R_{\text {tree }}$ & $0.193 / 0.226$ & $0.208 / 0.258$ & $0.163 / 0.189$ & $0.200 / 0.256$ & $0.2058 / 0.2491$ & $0.167 / 0.186$ & $0.209 / 0.235$ & $0.204 / 0.248$ & $0.191 / 0.240$ \\
\hline \multicolumn{10}{|l|}{ No. atoms } \\
\hline Protein & 4620 & 2287 & 1088 & 6943 & 4518 & 2407 & 2299 & 4498 & 4564 \\
\hline Ligand/ion & - & - & - & & - & - & - & - & - \\
\hline Water & 595 & 84 & 184 & 776 & 226 & 142 & 333 & 131 & 407 \\
\hline \multicolumn{10}{|l|}{$B$-factors } \\
\hline Protein & 36.4 & 49 & 33.5 & 49 & 38 & 37.9 & 30.9 & 45.4 & 32 \\
\hline Ligand/ion & - & - & - & - & - & - & - & - & - \\
\hline Water & 42.1 & 41.8 & 44.6 & 47 & 36.5 & 45.97 & 40.9 & 41.9 & 34.9 \\
\hline \multicolumn{10}{|l|}{ R.m.s. deviations } \\
\hline Bond lengths ( $(\AA)$ & 0.005 & 0.002 & 0.01 & 0.002 & 0.003 & 0.009 & 0.003 & 0.002 & 0.003 \\
\hline Bond angles $(Y)$ & 1.09 & 0.59 & 1.34 & 0.48 & 0.68 & 1.246 & 0.77 & 0.52 & 0.77 \\
\hline
\end{tabular}




\begin{tabular}{ccccc}
\hline Strains & Spoke/Cartwheel & Triplet/Centriole & Spoke/Triplet & $\mathbf{n}$ \\
\hline bld12 & ND & $8.88 \pm 0.69$ & ND & 137 \\
CrSAS-6 & $9.00 \pm 0.00$ & $9.00 \pm 0.00$ & $1.00 \pm 0.00$ & 24 \\
CrNN24 & $8.72 \pm 0.46$ & $8.87 \pm 0.34$ & $0.98 \pm 0.04$ & 39 \\
CrNN24/ $\Delta$ C2 & $7.08 \pm 0.86$ & $8.46 \pm 0.78$ & $0.84 \pm 0.08$ & 13 \\
CrNN24/ $\Delta$ N3 & $7.95 \pm 1.00$ & $8.45 \pm 0.60$ & $0.94 \pm 0.08$ & 22 \\
$\Delta$ C2 & $9.00 \pm 0.00$ & $9.00 \pm 0.00$ & $1.00 \pm 0.00$ & 10 \\
$\Delta$ N3 & $9.00 \pm 0.00$ & $9.00 \pm 0.00$ & $1.00 \pm 0.00$ & 11
\end{tabular}

